Running Head: CHANGE IN DEFENSIVE FUNCTIONING

\title{
CHANGE IN DEFENSIVE FUNCTIONING OVER THE COURSE OF GROUP PSYCHODYNAMIC INTERPERSONAL PSYCHOTHERAPY FOR WOMEN WITH BINGE EATING DISORDER
}

\author{
By \\ Robert P. Hill \\ A thesis submitted to \\ the Faculty of Graduate and Postdoctoral Affairs \\ in partial fulfillment of the requirements for the degree of \\ Master of Arts \\ in \\ Psychology \\ Carleton University \\ Ottawa, Canada
}

(C)2012 Robert P. Hill 


\title{
CHANGE IN DEFENSIVE FUNCTIONING
}

\begin{abstract}
This study examined change in defensive functioning during group psychodynamic interpersonal psychotherapy (GPIP) for women with Binge Eating Disorder (BED). Women with BED $(N=102)$ received 16-weeks of GPIP. Participants were assigned to a high or low attachment anxiety condition based on pre-treatment attachment anxiety scores. Three group therapy sessions (sessions 3, 8, and 16) from each of 12 groups were video recorded, transcribed, and participants were rated on an observer rating scale of defensive functioning, the Defense Mechanism Rating Scale. Defensive functioning was reliably assessed in this group therapy context and improved significantly during treatment. Change in defensive functioning was significantly associated with better selfesteem and lower interpersonal problems but only for women with high attachment anxiety. GPIP to improved defensive functioning, and change in defensive functioning for women with BED and high attachment anxiety is an important aspect for improving their self esteem and interpersonal problems.
\end{abstract}




\section{CHANGE IN DEFENSIVE FUNCTIONING}

\section{Acknowledgements}

First, I would like to thank my supervisor, Dr. George Tasca, whose support and feedback were immensely important in completing this thesis. I would also like to thank my co-supervisor, Dr. Mary Gick, and committee members, Dr. John Zelenski and Dr. Cecilia Taiana, for their time and feedback. Thank you to all of the project members who made this possible: Dr. Kylie Francis, Dr. Nancy McQuaid, Dr. Melissa Palardy, Renee Grenon, and Genevieve Hayden. Without their hard work and enthusiasm this project would never have got off the ground. Thank you to Dr. Michelle Presniak for your support and training during this past year. To all the members of the lab both at The Ottawa Hospital and Carleton University, thank you for your help and advice over the past two years. I would also like to thank my family for their endless support. Finally, I would like to thank Rae, who was with me through all the long days and late nights and who was always positive- even through the long presentations. 


\section{CHANGE IN DEFENSIVE FUNCTIONING}

Table of Contents

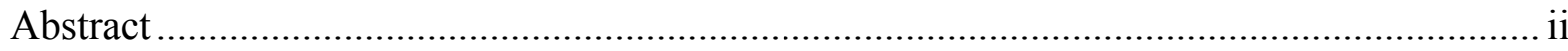

Acknowledgements ................................................................................................. ii

List of Tables ….................................................................................................. vii

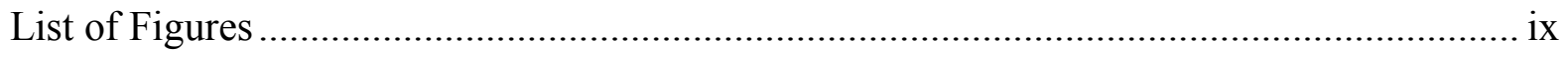

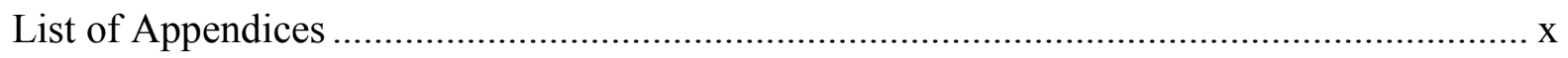

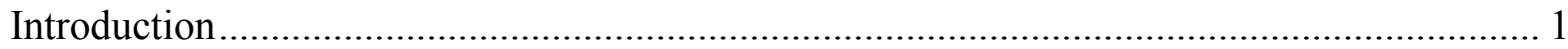

Defence Mechanisms and Defensive Functioning.............................................. 2

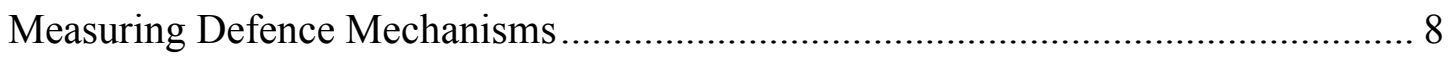

Binge Eating Disorder and Defensive Functioning ......................................... 10

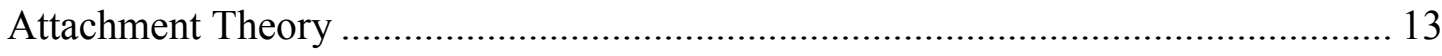

Group Psychodynamic Interpersonal Psychotherapy …..................................... 18

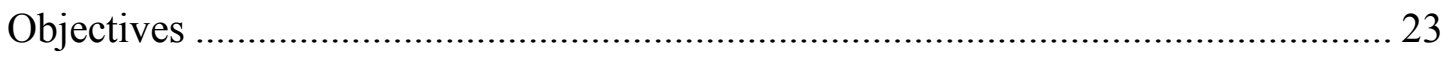

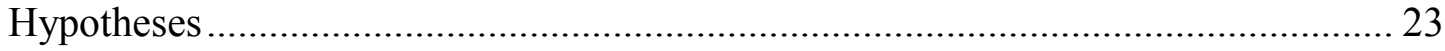

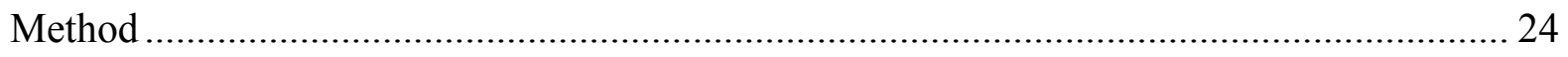

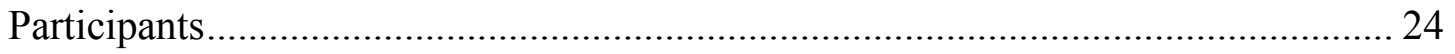

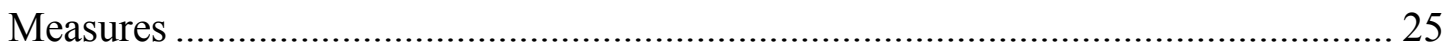

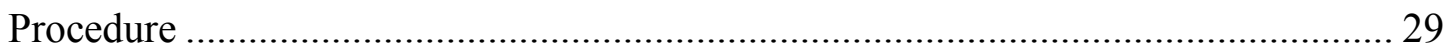

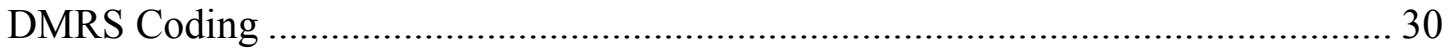

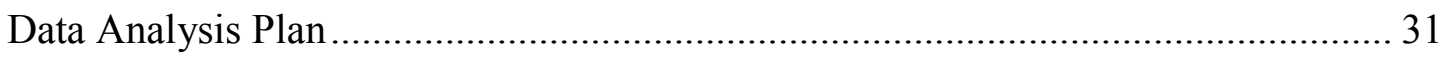

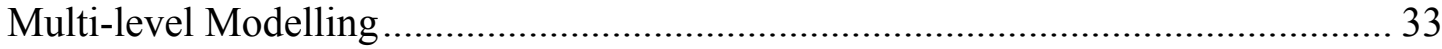

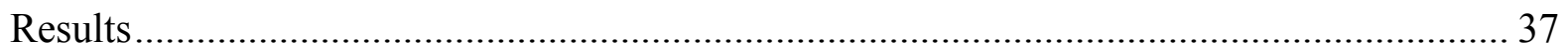




\section{CHANGE IN DEFENSIVE FUNCTIONING}

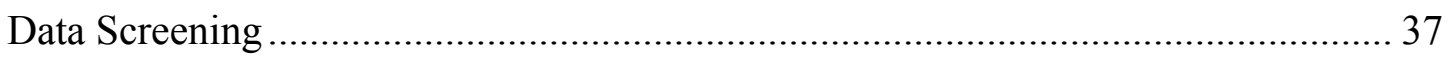

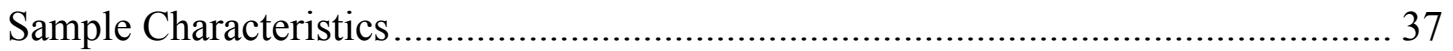

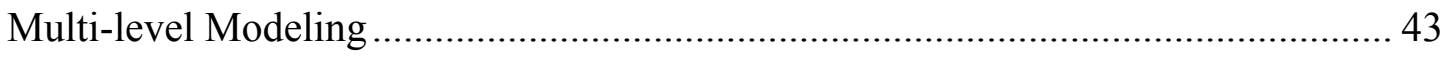

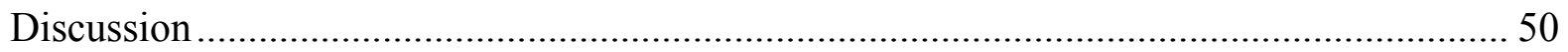

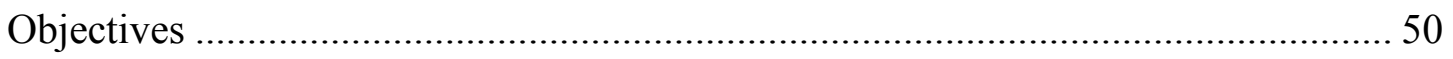

Reliability Between Raters Using the DMRS ............................................... 51

Overall Defensive Functioning and Treatment Outcome ..................................... 52

Change in Defensive Functioning During Group Treatment................................... 52

Change in Defensive Functioning Associated with Change in Treatment

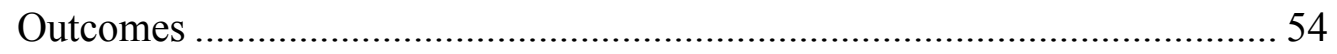

Change in Defensive Functioning and Change in Treatment Outcomes Moderated

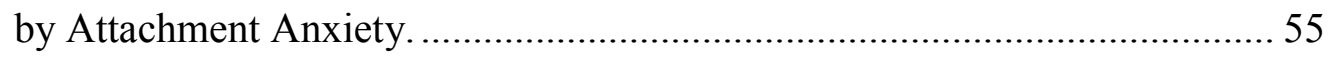

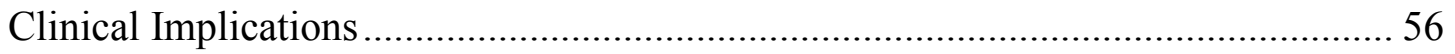

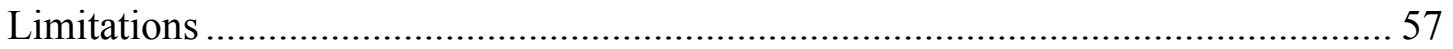

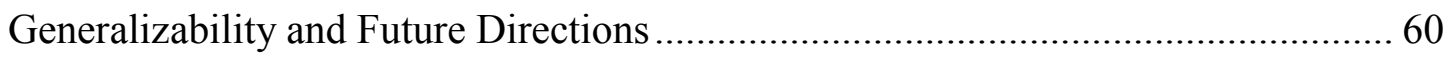

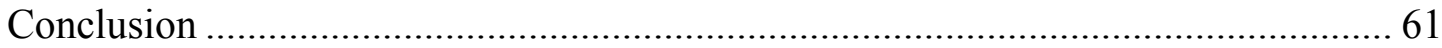

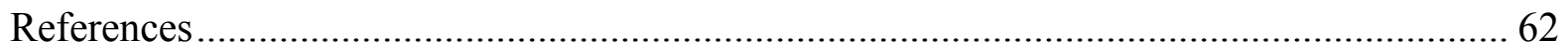

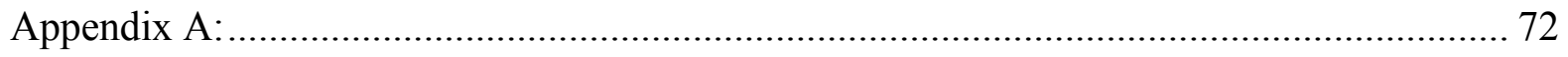

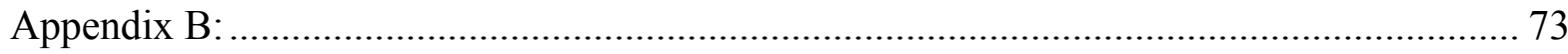

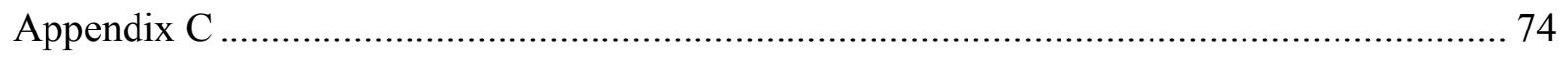

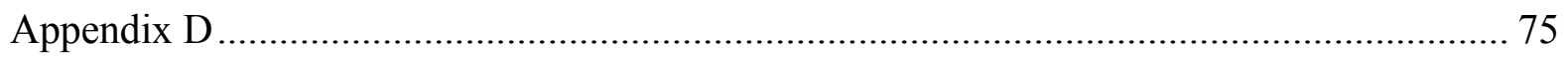

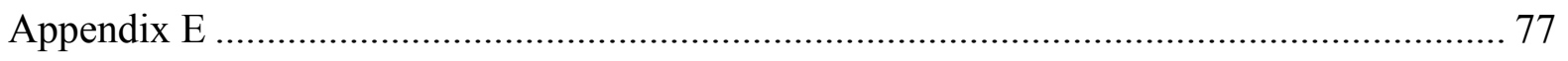




\section{CHANGE IN DEFENSIVE FUNCTIONING}

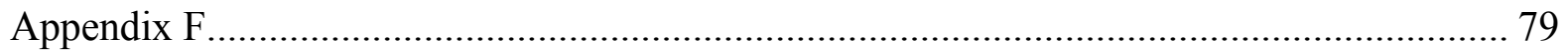

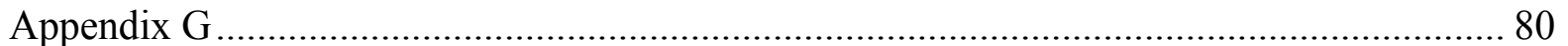




\section{CHANGE IN DEFENSIVE FUNCTIONING}

\section{List of Tables}

Table 1: List of defences and associated function from the DMRS

Table 2: Mean, Standard Deviation, and Range of the Number of Coded Defences by

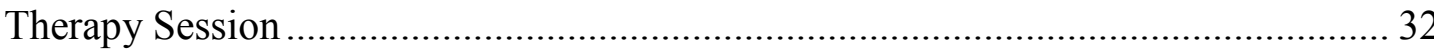

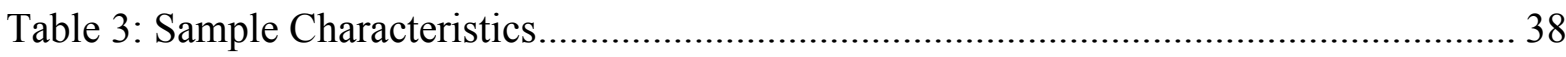

Table 4: Defence level ICC Values and Total Number of Defences Coded Between

Rating Pairs.

Table 5: Mean and Standard Deviations for Attachment Style Questionnaire Subscales at

Pre-treatment and Post-treatment by Attachment Anxiety Condition

Table 6: Correlations Between Session Three ODF Scores and Pre-treatment Measures of Depression, Interpersonal Problems, Self Esteem, and Binge Frequency

Table 7: Correlations Between Session 3 ODF Scores with Pre-treatment Subscales from the Attachment Styles Questionnaire.

Table 8: Correlations Between Session 16 ODF Scores with Post-treatment Measures of

Depression, Interpersonal Problems, Self Esteem, and Binge Episodes

Table 9: Correlations Between Session 16 ODF Scores with Post-treatment Subscales

from the Attachment Styles Questionnaire

Table 10: Mean and SD Values for ODF Scores at Sessions 3, 8, and 16 45

Table 11: Mean and SD for Treatment Outcome Variables from Pre-treatment to 12-

Months Post-treatment

Table 12: Multilevel Modelling of Change in Overall Defensive Functioning, Depressive Symptoms, Interpersonal Problems, Self Esteem and Binge Frequency Over Treatment 


\section{CHANGE IN DEFENSIVE FUNCTIONING}

Table 13: Change in Overall Defensive Functioning Scores Predicting Change in Treatment Outcome on Measures of Depression, Interpersonal Problems, Self

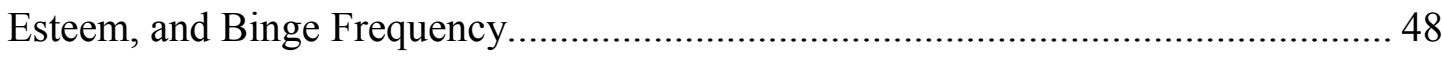

Table 14: 2-Level Model Results Testing the Moderating Effect of Attachment Anxiety Condition on the Relationship Between Change in Overall Defensive Functioning and Change in Treatment Outcomes.

Table 15: A 2-Level Model Testing Change in Overall Defensive Functioning and Change in Interpersonal Problems and Self-Esteem Outcomes by Treatment Condition. 


\title{
CHANGE IN DEFENSIVE FUNCTIONING
}

\author{
List of Figures
}

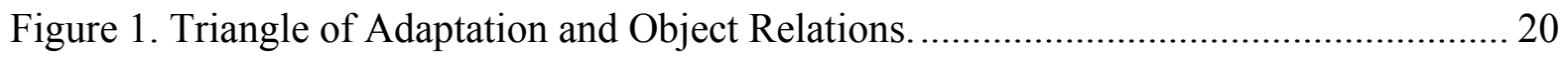




\section{CHANGE IN DEFENSIVE FUNCTIONING}

\section{List of Appendices}

Appendix A:Defence Mechanism Rating Scale Scoring Sheet

Appendix B:DSM-IV-TR Diagnostic Criteria for Binge-Eating Disorder .......................... 73

Appendix C: Attachment Style Questionnaire................................................................. 74

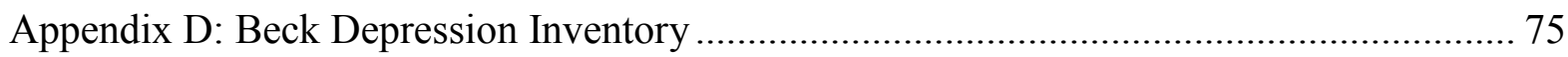

Appendix E: Inventory of Interpersonal Problems ................................................. 77

Appendix F: Rosenberg Self-Esteem Scale _................................................................. 79

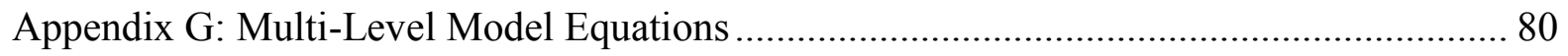


Change in Defensive Functioning Over the Course of Group Psychodynamic Interpersonal Psychotherapy for Women with Binge Eating Disorder

Freud first conceptualized defense mechanisms over a century ago(Vaillant, 1994). While the number and function of identified defences has changed, their impact on individualwell-being, on treatment, and on positive life outcomes remains important. The current study is the first to examine defensive functioning in women with Binge Eating Disorder (BED) undergoing group treatment. The first objective of the study is to establish the reliability and validity of an observer-based measure used to code defensive functioning in group therapy. The second objective is to examine if defensive functioning changes in response to group treatment (i.e. to a more adaptive level of defence). The third objective is to evaluate if a change in defensive functioning is associated with change in treatment outcomes. The fourth objective is to examine if the relationship between change in defensive functioning and change in treatment outcomes differs based on level of attachment anxiety.

Defence mechanisms are defined as "automatic psychological processes that protect the individual against anxiety and from the awareness of internal or external dangers or external stressors" (DSM-IV; American Psychiatric Association, 2000 p. 807). These automatic processes are typically unconscious mental operations, although in some situations they border on conscious awareness (Perry, Beck, Constantinides, \& Foley, 2009). Defence mechanisms are adaptive processes that work to manage internal conflict and stress by altering how these events are perceived. More adaptive defences typically maximize awareness of internal states and positive outcomes, whereas less adaptive defences work to restrict awareness of internal states and conflict, thus limiting positive 
outcomes (Perry et al., 2009; Vaillant, 1994). The pattern of defence mechanism use is referred to as defensive style (Vaillant, 1994). The relative use of adaptive defences compared to maladaptive defences is referred to as level of defensive functioning (Bond, 2004;Hersoug, Sexton, \&Høglend, 2002). Below, I will discuss specific defence mechanisms and their measurement.

\section{Defence Mechanisms and Defensive Functioning}

History of defence mechanisms. Sigmund Freud first conceived of defence mechanisms through his work with patients treated for hysteria (Cooper, 1998). He noticed that patients experienced a desire to keep their unpleasant symptoms, obsessions, and phobias out of their conscious awareness. He theorized that the defences were a means to balance the various forces (instinctual and emotional) acting on an individual. When the various structures of consciousness are out of balance a person will experience conflict and anxiety. This conflict and anxiety is managed through the use of a defence mechanism (Vaillant, 1994).

Anna Freud, building on the work of her father, published the Ego and the Mechanisms of Defence (1936/1966) which catalogued, labeled, and described the role of various defence mechanisms. Anna Freud synthesized work on defence mechanisms into a coherent framework (Vaillant, 1977) and classified defences based on the source of the anxiety that elicited a defence response (Cooper, 1998). She also described defence mechanisms as separate strategies to manage conflict, and presented the idea that some defence mechanisms can be advantageous to an individual (Vaillant, 1994). Furthermore, Anna Freud categorized defence mechanisms on a rudimentary hierarchy. She believed that Repression was the most effective defence, and that the other defences were utilized 
when Repression was unable to mask all conflict or manage anxiety (A. Freud, 1936/1966).

Hierarchy of Defence Mechanisms. Defence mechanisms are typically arranged on a hierarchy and ordered based on adaptive function and psychological health, and range from adaptive to maladaptive. Individual defences are typically clustered together into defence levels based on adaptive function and conceptual characteristics (Constantinides\& Beck, 2010). Vaillant (1977) organized 18 individual defence mechanisms into 4 broad categories arranged on a scale of adaptive functioning from: psychotic, immature, neurotic, to mature (Vaillant, 1977). In the Defensive Style Questionnaire (Andrews, Singh \& Bond, 1993), a commonly used self-report measure, individual defences are arranged in four clusters of highly correlated defence mechanisms. These defences are ranked on a continuum from least to most adaptive, comprised of: action patterns, image distorting, self-sacrificing, and adaptive patterns (Mullen, 1999; Bond, 2004).

The categorization and hierarchical classification are also reflected in the Defence Mechanisms Rating Scale (DMRS), an observer rated measure of defence mechanism use and function (Perry, 2009). The fifth edition of the DMRS (Perry, 1990) categorizes individual defences into 7 levels arranged from least adaptive to most adaptive (see Table 1). The DMRS is the parent measure to the DSM-IV Defensive Functioning Axis (Perry, Høglend, Shear et al., 1998). Each level contains defences with a common function, but a different method for handling conflict. For example, there are three individual defences clustered within the Disavowal Level, and each of these works to avoid unpleasant affect. Denial completely avoids the affect, Rationalization uses reassuring but incorrect 
explanations to remove awareness of the affect, and Projection avoids conflict by

projecting it onto others (Perry, 1990; Perry 2009).

Table 1

List of defences and associated function from the DMRS

\begin{tabular}{|c|c|c|}
\hline Level & Defence & Function \\
\hline \multirow{8}{*}{ 7. High Adaptive } & Affiliation & $\begin{array}{l}\text { Individual manages conflict or stressors by seeking } \\
\text { out support from others }\end{array}$ \\
\hline & Altruism & $\begin{array}{l}\text { Individual manages conflict or stressors by fulfilling } \\
\text { the needs of others }\end{array}$ \\
\hline & Anticipation & $\begin{array}{l}\text { Conflict or stress managed by considering realistic } \\
\text { or alternative solutions to future problems }\end{array}$ \\
\hline & Self-Assertion & $\begin{array}{l}\text { Conflict or stressor managed through the expression } \\
\text { of thoughts, feelings or wishes }\end{array}$ \\
\hline & Humor & $\begin{array}{l}\text { Emotional conflict or stress managed by } \\
\text { emphasizing amusing or ironic aspect of conflict }\end{array}$ \\
\hline & Self-Observation & $\begin{array}{l}\text { Emotional conflict or stress managed by reflecting } \\
\text { on own thoughts, feelings, and behaviours }\end{array}$ \\
\hline & Sublimation & $\begin{array}{l}\text { Emotional conflict or stress managed by } \\
\text { channelling feelings into socially acceptable } \\
\text { behaviours }\end{array}$ \\
\hline & Suppression & $\begin{array}{l}\text { Emotional conflict reduced by consciously avoiding } \\
\text { thinking about problem }\end{array}$ \\
\hline \multirow{3}{*}{ 6. Obsessional } & Isolation & $\begin{array}{l}\text { Emotional conflict or stress reduced by separating } \\
\text { cognitive and affective components of the } \\
\text { experience/conflict }\end{array}$ \\
\hline & Intellectualization & $\begin{array}{l}\text { Emotional conflict or stress is managed by use of } \\
\text { abstract thought }\end{array}$ \\
\hline & Undoing & $\begin{array}{l}\text { Emotional conflict or stress is managed through } \\
\text { behaviour designed to make amends for negative } \\
\text { thoughts and feelings }\end{array}$ \\
\hline \multirow{5}{*}{ 5. Other Neurotic } & Displacement & $\begin{array}{l}\text { Emotional conflict or stress is managed by moving } \\
\text { feelings in response to an object to another, less } \\
\text { threatening object }\end{array}$ \\
\hline & Reaction & Emotional conflict is managed by substituting \\
\hline & Formation & $\begin{array}{l}\text { difficult thoughts or feelings with the opposite } \\
\text { thoughts or feelings }\end{array}$ \\
\hline & Dissociation & $\begin{array}{l}\text { Emotional conflict is managed by altering their } \\
\text { conscious state and identity }\end{array}$ \\
\hline & Repression & $\begin{array}{l}\text { Emotional conflict is managed by being unable to } \\
\text { remember or be cognitively aware of their affective } \\
\text { states or experiences }\end{array}$ \\
\hline $\begin{array}{l}\text { 4. Minor Image } \\
\text { Distorting }\end{array}$ & Omnipotence & $\begin{array}{l}\text { Emotional conflict is reduced by acting superior to } \\
\text { others, or belief in having special-powers }\end{array}$ \\
\hline
\end{tabular}




\begin{tabular}{|c|c|c|}
\hline \multirow[t]{3}{*}{ (Narcissistic) } & Idealization & $\begin{array}{l}\text { Emotional conflict or stress is managed by } \\
\text { attributing overly positive qualities to self or others }\end{array}$ \\
\hline & Devaluation & Emotional conflict or stress is managed by \\
\hline & Fantasy & $\begin{array}{l}\text { attributing overly negative qualities to self or other } \\
\text { Emotional conflict or stress is managed through } \\
\text { excessive daydreaming }\end{array}$ \\
\hline \multirow{3}{*}{ 3. Disavowal } & Rationalization & $\begin{array}{l}\text { Emotional Conflict or stress is managed through } \\
\text { reassuring but incorrect explanations of self or } \\
\text { others behavior }\end{array}$ \\
\hline & Projection & $\begin{array}{l}\text { Emotional Conflict or stress is managed by falsely } \\
\text { attributing ones own emotional state onto others }\end{array}$ \\
\hline & Denial & $\begin{array}{l}\text { Emotional conflict or stress is managed by refusing } \\
\text { to acknowledge reality of one's experience }\end{array}$ \\
\hline \multirow{2}{*}{$\begin{array}{l}\text { 2. Major Image } \\
\text { Distorting } \\
\text { (Borderline) }\end{array}$} & $\begin{array}{l}\text { Projective } \\
\text { Identification }\end{array}$ & $\begin{array}{l}\text { Emotional conflict or stress is managed by } \\
\text { projecting that state onto someone else and a belief } \\
\text { that the state originated with the other person }\end{array}$ \\
\hline & Splitting & $\begin{array}{l}\text { Conflict or stress managed by viewing self or other } \\
\text { as all good or all bad and failing to integrate other } \\
\text { states }\end{array}$ \\
\hline \multirow{3}{*}{ 1. Action } & Hypochondriasis & $\begin{array}{l}\text { Repetitious use of a complaint used to ask for help } \\
\text { from others, characterized by consistent rejection } \\
\text { and covert feelings of hostility towards others for } \\
\text { being unable to help }\end{array}$ \\
\hline & $\begin{array}{l}\text { Passive- } \\
\text { Aggression }\end{array}$ & $\begin{array}{l}\text { Conflict or stress managed by indirectly and } \\
\text { unassertively expressing aggression towards others }\end{array}$ \\
\hline & Acting Out & $\begin{array}{l}\text { Acting without reflection or with a disregard to any } \\
\text { negative consequences }\end{array}$ \\
\hline
\end{tabular}

Note. Defence Levels range from 1 to 7 . Higher scores indicate more adaptive Overall Defensive Functioning (ODF) and lower scores indicate less adaptive functioning (Perry et al., 2009). DMRS = Defence Mechanism Rating Scale.

Defences across lifespan. Defence mechanism use is present in both children as well as adults (Cramer, 2007). Different defences may be used at different periods in one's lifetime as an individual moves through developmental stages (Cramer, 2007). As children move into adolescence and adulthood, they will likely utilize more cognitively complex defences. In early childhood, Denial is the most often used defence, followed by Projection in adolescence, then more adaptive defences in later life (see Table 1). Similarly, Vaillant (1977) reported that adults from the longitudinal Grant Study who relied on more "immature defences" (e.g. Projection) in adulthood, had the poorest 
outcomes in terms of happiness, career success, and marital satisfaction (p. 158-161). In a separate study conducted by Vaillant, 369 boys were followed for 35 years. He found that use of adaptive defensive styles (such as Altruism and Suppression, see Table 1) were highly correlated with good mental health. Conversely, maladaptive styles (Hypochondriasis, Passive Aggression, and Dissociation; see Table 1) were associated with impaired mental health (Vaillant, 1994). Cramer (2012) presented the results of two longitudinal studies that examined change in the use of defence mechanisms from childhood to adulthood and found that maturity, indicated by scores on a measure of ego strength (which is associated with the ability to use one's resources to effectively adapt to new internal and external events), predicted the use of more adaptive defence mechanisms (Cramer, 2012).

Defensive functioning, psychopathology and psychotherapy.Perry and Cooper (1989) examined adaptive defensive functioning of clinical interviews and life vignettes in 76 patients undergoing treatment for borderline personality disorder. The authors demonstrated that lower levels of adaptive defensive functioning were associated with higher reported symptom levels of depression, anxiety, mania, and greater impairment in psychological functioning at the time of treatment. Perry and Cooper (1989) also reported that maladaptive defence mechanism use was predictive of poorer outcomes on measures of global and psychosocial functioning for those seeking treatment for depression (Perry \& Cooper, 1989 Lower adaptive defensive functioning was associated with greater reported symptoms of depression and anxiety, poorer interpersonal functioning, and greater global levels of distress in a sample of patients with various illnesses seeking individual therapy in an outpatient clinic (Hilsenroth, Callahan, \&Eudell, 2003). 
Adaptive defensive functioning is associated with a reduction in symptom distress and more positive treatment outcomes for patients undergoing psychotherapy treatment. For example,Høglend and Perry (1998) found that greater use of defences from the lower half of the DMRS hierarchy (i.e. more maladaptive defences) predicted lower scores on measures of symptom severity when measured six months after starting individual therapy for patients suffering from a variety of mental illnesses. Higher defensive functioning, specifically the use of Self-Observation, predicted better outcomes six months after the start of individual therapy, (Høglend\& Perry, 1998). In a study of 17 patients undergoing Group Behavioural Therapy for Obsessive Compulsive Disorder, Albucher, Abelson, \&Nesse (1998) reported that adaptive defence mechanism use increased after treatment, which was related to a decrease in scores on a measure of obsessive compulsive behaviour, as well as decrease in depression scores. The change to more adaptive defence mechanism use was maintained at one-year post-treatment (Albucher, Abelson, \&Nesse, 1998). compulsive behaviour, as well as decrease in depression scores. The change to more adaptive defence mechanism use was maintained at when measured one-year post-treatment (Albucher, Abelson, \&Nesse, 1998).

In a more recent study by Johansen et al. (2011), 50 patients who met the diagnostic criteria for Cluster C Personality Disorder (e.g. Avoidant, ObsessiveCompulsive, Passive Aggressive, and Dependent) were randomized into short term Dynamic Therapy or short term Cognitive Behaviour Therapy. The results showed that increases in adaptive defensive functioning predicted lower levels of distress, measured by the Symptom Checklist-90, from pre-treatment to 2 years post-treatment for both interventions (Johansen et al., 2011). The results also indicated that change in ODF 
during treatment in both therapy conditions predicted lower symptom distress posttreatment, which was stable up to two years post-treatment (Johansen et al., 2011).

Finally, Presniak et al., (2010) presented data from a case study of a 50-year old man undergoing three years of psychoanalytic therapy. The authors found that over the course of treatment defensive functioning scores for the patient improved, indicating greater use of adaptive defences. The case study reinforces the research presented earlier in this section that found that increases in defensive functioning is related to positive treatment outcomes, which are maintained after treatment ends.

\section{Measuring Defence Mechanisms}

Self-report vs. observer-report.Assessing defence mechanisms is a challenge for researchers because they are inferred psychological processes of cognitive and mental constructs. The challenge is exacerbated by the lack of consensus among researchers regarding the order and number of defences (Bond, 2004). Measurement tools for defence mechanisms generally fall into two categories: observer-report, and self-report. Selfreport measures of defence mechanisms are widely used and can be distributed to a large sample quickly. A major limitation of self-reports is the unconscious nature of defence mechanisms. That is, because a defence mechanism is a mental operation typically outside of awareness, and self-reports require awareness, using a self-report measure may not accurately indicate the presence of a defence mechanism. However, self-report measures such as the DSQ are widely used (Bond, 2004). The DSQ likely provides indirect evidence of use of a defence, and so can only really claim to be measuring the conscious derivative of a defence (Bond, Perry, Gautier, Goldenberg, Oppenheimer, \&Simand, 1989; Perry \& Cooper, 1989). When compared to self report measures, 
observer rated measures of defence mechanisms are more time consuming, but may demonstrate greater validity because the observer relies on inferences and judgement to arrive at a conclusion (Perry \&Ianni, 1998).

Defence Mechanism Rating Scale. There are several different interview-based rating scales of defence mechanisms; however the current study will focus on the Defence Mechanism Rating Scale (DMRS). The DMRS is a widely used observer rating measure developed by Chris Perry (Hersoug, Sexton, \&Høglend, 2002; Mullen, 1999). The DMRS categorizes 27 defences separated into 7 summary defence scales or levels. The summary categories were created by combining highly correlated individual defences. Summary levels are arranged on a continuum from Maladaptive to High Adaptive defences, rated from 1 to 7 , respectively (see Table 1). The higher level defences (e.g., High Adaptive) are associated with better psychological functioning, while the lower level defences (e.g. Action) are associated with greater psychopathology (see Table 1; Perry \& Cooper, 1989).

Defensive functioning measured by the DMRS, is a distinct, albeit slightly correlated, construct from measures of symptom distress, anxiety, and depression (Perry \&Høglend, 1998). Hilsenroth, Callahan and Eudell (2003) tested the convergent and discriminant validity of observer based ODF scores based on the DMRS in a population of outpatient clients undergoing psychotherapy. The results found convergent validity between the ODF scores rated by clinicians and self-report measures of psychopathology (e.g. depression, interpersonal problems, and anxiety). Specifically, lower ODF scores were associated with greater self-report symptoms of psychopathology and distress, but they were only moderately correlated. The authors concluded that ODF scores are part of 
a construct distinct from self-report measures of psychopathology and distress (Hilsenroth, Callahan, \&Eudell, 2003).

As indicated above, studies using the DMRS have examined a range of populations, including diagnosis free populations, individuals diagnosed with personality disorders, such as borderline personality disorder and anti-social personality disorder (Presniak et al., 2009), and patients seeking treatment for depression (Perry \&Høglend, 1998). The current study will be the first to use the DMRS obtained from a sample of women diagnosed with an eating disorder, specifically in a sample diagnosed with Binge Eating Disorder.

\section{Binge Eating Disorder and Defensive Functioning}

Binge Eating Disorder.Binge Eating Disorder (BED) is classified as an Eating Disorder Not Otherwise Specified (EDNOS) and the diagnostic criteria for BED were first included in appendix B of the DMS-IV TR (APA, 2000; see also Appendix B of this thesis). BED is now a full diagnosis in DSM-5 (APA, 2013), although for this study the DSM-IV criteria and definition was used. BED is characterized by recurrent binge eating, feelings of shame or guilt, self-evaluation that is unduly negatively influenced by weight and shape, but with no compensatory behaviour (e.g. vomiting, laxative use and overexercise). In BED binge eating, (i.e., consuming a large amount of food in a discrete period of time with a sense of loss of control) occurs twice a week for at least 6 months. Bulimia Nervosa has similar characteristics to BED but those with Bulimia Nervosa often engage in inappropriate compensatory behaviours (e.g. laxative use, vomiting, and exercise) following a binge (DSM IV, APA, 2000) 
A diagnosis of BED is associated with a higher body mass index (BMI; a measure of body mass calculated by dividing weight in kilograms by height in meters squared) compared to individuals diagnosed with Bulimia Nervosa (Striegel-Moore et al., 2001) or individuals not diagnosed with an eating disorder (Kessler et al., 2012). Women with $\mathrm{BED}$, compared to those not diagnosed with BED, often report higher lifetime prevalence of major depression, anxiety disorders, such as panic disorder and phobias (Bulik, Sullivan, \&Kendler, 2002), as well as lower self-esteem and body dissatisfaction than individuals of similar weight (Striegel-Moore, Wilson, Wilfley, Elder, \& Brownell, 1998). BED is also co-morbid with a host of physical conditions, including pain conditions, diabetes, hypertension, ulcers, and gastrointestinal problems (Bulik et al., 2002; Kessler et al., 2012).

A recent Canadian survey of approximately 1500 adult women examining the prevalence of eating disorders found that BED was the most commonly reported eating disorder $(3.8 \%, 95 \% \mathrm{CI}=3.7-3.9 \%)$ compared to Anorexia Nervosa (restricting and binge-purge subtypes) $(<.1 \%)$ and Bulimia Nervosa (purge subtype) $(0.2 \%, 95 \% \mathrm{CI}=$ .18-.22). The survey further reported that mean and median age of onset for BED was 23.33 years. Of women with BED, $57.7 \%$ received lifetime treatment for emotional problems, though only $38.3 \%$ of lifetime cases of BED received treatment designed specifically for eating disorders (Kessler et al., 2012).

Eating disorders and defensive styles. There are several studies that have examined the relationship between eating disorders (specifically Anorexia and Bulimia Nervosa) and adaptive defensive functioning. The results consistently indicate that an eating disorder diagnosis is associated with greater maladaptive defensive style compared 
to non-eating disordered controls (see Bond, 2004 for review), including immature defences, such as Undoing, Passive Aggression, and Acting Out (Blaase\&Elklit, 2001; Poikolainen et al., 2001). Furthermore, an eating disorder diagnosis is associated with a greater use of a neurotic defensive style (Stein, Bronstein, \&Weizman, 2003).

To date there has not been a single study conducted on the defensive functioning of those diagnosed with BED. Therefore, it is difficult to predict defence mechanism use and the defensive functioning prevalent within that population. Theoretically, one could argue that a binge episode functions in much the same way as a defensive strategy or even a coping strategy. The function of a binge may be to reduce feelings of anxiety and conflict that the individual is feeling (Tasca, Mikail, \& Hewitt, 2005). The binge then works to suppress those unpleasant emotions by consuming a large amount of food. A binge could be considered a coping strategy, since in many cases, individuals with BED report entering a dissociated state when starting a binge as well as a compulsion to eat until all the food is gone (Fairburn, 1995).

According to the Interpersonal Model of BED, interpersonal problems produce negative affect, which then leads to episodes of disordered eating (Ansell, Grilo, \& White, 2012). The Interpersonal Model view of eating disorders is similar to the psychodynamic view of binge eating, which posits that feelings of anxiety and conflict are produced by unmet attachment needs, which leads to a binge episode as a method to reduce those negative feelings (Tasca, Mikail, \& Hewitt, 2002). In both the Interpersonal Model and the psychodynamic view, a relational deficit produces negative affect which triggers a compensatory behaviour, characterized by a loss of control for those with BED 
(Ansell et al., 2012); this pattern is closely related to current conceptualizations of a defence mechanism.

\section{Attachment Theory}

As previously mentioned, binge eating likely functions to reduce negative feelings of conflict and anxiety. The conflict may be produced by unmet attachment needs (Tasca et al., 2002). Attachment theory outlines the basic process that people use to form emotional bonds with primary caregivers and, later in life, romantic partners. The need for attachment provides motivation for infants to seek closeness to attachment figures. According to the theory, children develop expectations about relationships through repeated interactions with their caregiver (Mikiluncer\& Shaver, 2007). How the child and caregiver interact becomes encoded in implicit memory, which forms internal working models of attachment (Tasca, Ritchie et al., 2011). Internal working models guide how a person will interact with their environment, regulate their affect and cope with stress (Hazan\& Shaver, 1987).

Bowlby introduced the concept of attachment theory in adults over fifty years ago. Bowlby's original theory described how infants become emotionally attached to their caregiver and how infants react when separated from their caregiver (Hazan\& Shaver, 1987). If the primary caregiver is perceived as warm, caring and validating of the child, then the child will likely form a secure attachment style. Conversely, if a child experienced a cold-dismissive, overbearing, or an inconsistent pattern of behaviour from the primary caregiver, then an insecure attachment style is likely to form (Mikiluncer\& Shaver, 2007). 
Insecure attachment can further be characterized as either anxious or avoidant. Individuals high in attachment anxiety engage in hyper-activating strategies which work to up-regulate emotions. These strategies function to maintain a high level of hypervigilance designed to allow the person to be on guard for potential loss or threats in a relationship (Tasca, Ritchie \& Balfour, 2010). This increased hyper-activation leads to greater efforts to elicit attention, protection, and concern from the primary attachment figure through insistent expressions of need on the part of the anxious individual (Maxwell et al., 2013; Mikiluncer\& Shaver, 2007).

Individuals high in attachment avoidance desire independence and self-reliance very intensely. They downplay relational/attachment needs and tend not to monitor the availability of attachment figures or any actual or potential threats to their relationship. Their de-activating strategy is characterized by a down regulation of emotions, which works to keep emotions and others at a distance. The distance created by down regulating allows them to avoid any reactivation of their attachment needs (Mikiluncer\& Shaver, 2007; Tasca, Ritchie, \& Balfour, 2010).

According to Harris (2004), children seek out relationships that reaffirm their internal working model, thus reciprocating and reinforcing the child's model through their environment (Harris, 2004). Research has found that attachment styles are relatively stable over time. For example, in one longitudinal study attachment assessed at infancy was predictive of attachment patterns assessed 20 years later, with $72 \%$ of participants showing no change in secure versus attachment patterns (Waters et al., 2000b). While often stable, attachment patterns can change. Individuals sometimes move between attachment styles, typically as a result of negative life events (e.g., divorce/loss of a 
parent, life threatening illness of a parent or child, or childhood sexual trauma) or positive life events (positive romantic relationship, psychotherapy; Bakermans-Kranenburg\& van Ijzendoorn, 2009; Waters et al., 2000a; Waters et al., 2000b).

Attachment and eating disorders. Attachment and eating pathology is a growing area of research, although there are fewer studies testing the relationship between attachment and BED. Research to date has found that among women diagnosed with eating disorders, higher levels of attachment insecurity are more prevalent compared to women with no eating disorder diagnosis (Illing et al., 2010). Attachment insecurity, specifically anxious and avoidant attachment can influence the severity of the disorder through the promotion of increased body dissatisfaction (Troisi et al., 2006). A previous study testing the association between attachment styles and eating symptoms and traits, found that preoccupied or anxious-ambivalent styles (scales that are moderately correlated with attachment anxiety) were a strong predictor of eating pathology (e.g., concern about body weight and shape, preoccupation with weight, and bulimia tendencies), while no association between attachment avoidance and eating pathology was found (Suldo\& Sandberg, 2000). In a more recent study, Koskina and Giovazolias (2010) found that attachment anxiety and attachment avoidance affected eating behaviours differently. The authors reported that the relationship between attachment anxiety and disordered eating was mediated by body esteem. However, no mediating effect of body esteem for the relationship between attachment avoidance and disordered eating. Attachment avoidance had a direct effect on disordered eating (Koskina\&Giovazolias, 2010). 
Illing et al. (2010) examined the impact of attachment insecurity on eating disorder symptoms and outcome following an intensive 14-week day hospital program. The results showed that women with an eating disorder diagnosis reported higher levels of attachment insecurity compared to a control group of women with no eating disorder diagnosis. Furthermore, higher scores on the Need for Approval subscale of the Attachment Styles Questionnaire (Feeney, Nollar, \&Hanrahan, 1994), an indicator of attachment anxiety, were associated with greater eating disorder symptoms measured at pre-treatment, whereas attachment avoidance subscales showed no significant relationship with symptom severity (Illing et al., 2010).

Tasca and colleagues (2006) examined whether attachment insecurity could predict treatment outcome in a randomized control trial comparing BED patients who received Group Cognitive Behavioural Therapy (GCBT) and Group Psychodynamic Interpersonal Psychotherapy (GPIP). The results of the trial found that higher attachment anxiety at pre-GPIP treatment was related to better outcomes at post-treatment, whereas lower attachment anxiety scores were associated with better progress in GCBT (Tasca et al., 2006). The authors speculated that GPIP, which focuses on affect regulation and relationship patterns may be particularly suited to those with BED and attachment anxiety. In a follow-up study comparing BED patients in a high or low attachment anxiety condition undergoing GPIP, Tasca et al (2013) found that for patients high in attachment anxiety, increases in therapeutic alliance during GPIP predicted better binge eating outcomes (Tasca et al, 2013).

Attachment and defensive functioning. Research on defensive functioning and attachment is sparse. In their review, Mikiluncer and Shaver (2002) report that 
attachment insecurity is related to greater use of Projection and Projective Identification. Specifically, those high in attachment anxiety typically project their own traits onto other people and strangers, often making incorrect assumptions about that person's behaviour and personality based on that Projection. The function of this behaviour is to maintain closeness in the relationship through increased affiliation. Those high in attachment avoidance project onto others; however, they typically project their self-perceived negative attributes onto others, which allows them to distance themselves from that person (Mikiluncer\& Shaver, 2002).

Research found that individuals high in attachment avoidance defensively exclude painful memories and keep them cognitively distanced (Mikulincer and Shaver, 2002). These individuals also tend to engage in self-inflation when dealing with threatening interpersonal situations (Mikiluncer and Shaver, 2002). Based on the DMRS hierarchy of defences, an individual high in attachment avoidance will likely engage in more MinorImage Distorting defences (e.g. Omnipotence, Devaluation of Self/Other, and Idealization; see Table 1) as well as Disavowal level defences (e.g. Denial, Rationalization, and Projection; see Table 1). Minor Image Distorting defences reduce conflict by altering the perception of that conflict by exaggerating positive or negative qualities about self or other, respectively. Disavowal level defences work to reduce affect by removing that conflict from awareness (Perry, 1990).

Conversely, those high in attachment anxiety will keep painful memories readily accessible to maintain a state of hyper-vigilance, and when faced with threatening interpersonal situations they tend towards self-devaluation (Mikiluncer\& Shaver, 2002). Individuals high in attachment anxiety might rely on defences at the Major Image 
Distorting level (see Table 1). These defences work to reduce feelings of conflict by viewing an individual as all bad or all good (Splitting), or by attributing unpleasant feelings to another person while still acknowledging those feelings (Projective Identification). When Splitting or using Projective Identification, the feelings of conflict are not disavowed but are held onto very strongly (Perry, 1990).

In the current study I will assess differences in defensive functioning between participants with BED who are higher versus lower in Need for Approval, an index of attachment anxiety. I predict that those in the high attachment anxiety condition will have lower ODF scores compared to individuals lower in attachment anxiety (i.e., those with higher in attachment avoidance and higher in attachment security). This study will be the first to test the association between DMRS defensive functioning and attachment anxiety in a population of women with BED. I expect the results to be consistent with Mikulincer\& Shaver's (2007) findings, particularly that ODF scores for participants higher in attachment anxiety will be associated with less adaptive defence levels (e.g. major image distorting) compared to ODF scores for participants low in attachment anxiety and attachment avoidance (e.g. minor image distorting and disavowal defences) and participants high in attachment security (e.g. high adaptive defences). Another goal of this study is to evaluate if defensive functioning as measured by the DMRS becomes more adaptive during group psychotherapy, and whether this change is associated with symptom improvement for those with BED receiving GPIP.

\section{Group Psychodynamic Interpersonal Psychotherapy}

Group Psychodynamic Interpersonal Psychotherapy (GPIP; Tasca et al., 2005) is a therapeutic method originally designed for treatment of patients with BED. GPIP 
combines theoretical aspects of psychodynamic, interpersonal, and group therapy theories. One important theory underlying GPIP is Malan's (1979) construct called the Triangle of Conflict and the Triangle of Person. Malan's model was adapted by Tasca and colleagues $(2002 ; 2005)$ to focus on Object Relations Theory. The triangles were renamed the Triangle of Adaptation and Triangle of Object Relations to describe the innate need to affiliate and seek out other people in close relationships (Figure 1). This need is similar to Bowlby's theory of attachment and highlights the importance of relationships on psychological well-being (Figure 1; Tasca, et al., 2002). The Triangle of Adaptation can be used to define binge eating as a defensive function; for example, unmet attachment needs may lead to negative affect that may require binge eating as a means of coping. The Triangle of Object Relations is useful for conceptualizing how a person relates to others in their environment and how past relationships and self-concepts shape current relationships. According to Tasca et al., (2002), the Triangle of Adaptation is conceptually embedded within the Triangle of Object relations, which influences how a person views past relationships (based on met or unmet attachment needs), current views of self or other (which can produce anxiety or negative affect), and their relationship to the therapist and group (which impacts how the person engages with the therapist and group; Tasca et al, 2002). 
Triangle of Adaptation

Defense

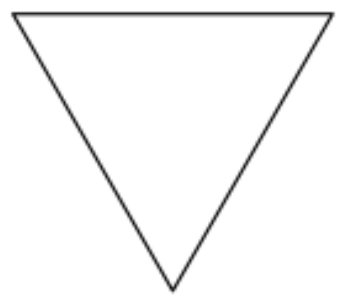

Attachment Needs
Triangle of Object Relations

Therapist/Group Current Self and Other

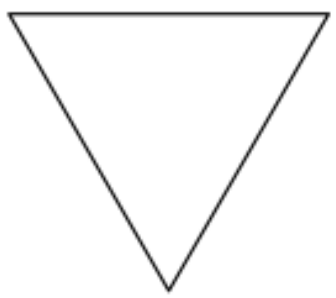

Past Relationship with Self and Other

Figure 1. Triangle of Adaptation and Object Relations. Model decribing how a person views their environment and self can influences their relationships with others(Tasca et al., 2002).

In GPIP, the patient is encouraged to explore more adaptive interpersonal patterns in a safe group environment. The Triangle of Object Relations illustrates how past relationships may be a template for current relationships with others or relationships in therapy groups. One of the main goals of GPIP is to shift maladaptive interpersonal interactions to more adaptive patterns. To do so, the therapist identifies the client's unique interpersonal pattern. When the therapist shifts the patients' interpersonal patterns, patients can feel anxiety or tension. In turn patients may engage in a defensive response that aims to re-engage in the original interpersonal pattern.

From the interpersonal perspective, the patients in therapy will seek out relationships and will typically engage in conversations and patterns of communication that will reinforce their self-concept, and interpersonal pattern. Patients engaging in GPIP are encouraged to use interpersonal patterns that differ from their typical style. The group is set up to allow patients to test these new interpersonal patterns in the therapy and to generalize the new patterns to interpersonal relationships in their everyday life (Tasca et 
al., 2002). By disrupting problematic interpersonal patterns and replacing them with more adaptive patterns, patients can satisfy their relational and attachment needs better, which will in turn, reduce negative affect and thus reduce the urge to binge (Ansell et al., 2012; Tasca et al., 2002).

In a randomized control trial testing the effectiveness of GCBT compared to GPIP in a sample of patients with binge eating disorder, both GCBT and GPIP resulted in a reduction of the number of days binged following treatment compared to a wait-list control measured at post-treatment and up to the 12 month follow up (Tasca et al., 2006). The randomized control trial also tested the association of pre-treatment attachment styles and change in number of days binged between the two treatment conditions. Participants in both treatment conditions displayed a reduction in the number of days binged; however, the results differed based on level of anxious attachment and treatment condition. Participants with high attachment anxiety scores showed a greater reduction in the number of days binged in GPIP, compared to those with low attachment anxiety. Participants in the CGBT condition with low attachment anxiety reported a greater reduction in the number of days binged, compared participants with high attachment anxiety. In other words, GPIP was more effective for participants with high attachment anxiety than low attachment anxiety on binge eating outcomes (Tasca et al., 2006).

A follow up study conducted by Tasca et al (2013) supported the effectiveness of GPIP in reducing episodes of binge eating, as well as increasing positive treatment outcomes. The study compared treatment outcomes for women with BED who were assigned to a high attachment anxiety condition or a low attachment anxiety condition based on pre-treatment attachment anxiety scores. The results indicated that patients in 
both the low and the high attachment anxiety condition reported fewer depressive symptoms, interpersonal problems, and episodes of binge eating, as well as an increase in self-esteem. Both conditions benefited from treatment; however, for patients in the high attachment anxiety condition, increases in therapeutic alliance during GPIP resulted in better binge eating outcomes (Tasca et al., 2013).

Therapeutic alliance describes the emotional bond and the agreement on goals and tasks for the group therapy between an individual member and the group or the therapist (Tasca et a., 2007). A previous treatment study comparing the relationship between therapeutic alliance and attachment anxiety in GPIP compared to GCBT found that positive treatment outcomes (reduction in the number of days binged) for patients with high attachment anxiety were related to increases in therapeutic alliance. The authors suggested that patients high in attachment anxiety benefitted more from GPIP's focus on interpersonal functioning and emotion regulation. GPIP led to attachment schemas being primed, and thus requiringa growth in therapeutic alliance in order to achieve positive outcomes (Tasca et al., 2007). The findings of both studies suggest that compared to those low in attachment anxiety patients high in attachment anxiety required greater agreement on tasks and goals and improved bonding among group members (Tasca et al., 2007; 2013).

GPIP and defensive functioning.Consistent with previous individual therapy research using the DMRS, we expect that participants in the current group therapy study will exhibit an adaptive change in defensive functioning from early to late group therapy sessions. Previous research also found positive change in adaptive defence mechanism use for patients after only seven weeks of individual therapy (Albucher, Abelson, 
\&Nesse, 1998), and that change was associated with lower symptom distress at post individual treatment (Johansen et al., 2011). Therefore, we predict that change in defensive functioning will be associated with treatment outcomes for women with BED receiving GPIP. Given that those with high attachment anxiety will likely engage in lower defensive functioning, we also predict that the change in defensive functioning outcome relationship will be greater in the high attachment anxiety condition.

\section{Objectives}

The first goal of this study is to establish adequate reliability and validity of the DMRS in a group setting. This is important since the DMRS has never been utilized in a group therapy setting. The second objective is to examine change in defensive functioning over the course of 16 sessions of group therapy. The third objective will be to assess associations between change in adaptive defensive functioning and symptom outcome for women with BED receiving group therapy. The fourth objective is to examine whether the relationship between change in adaptive defensive functioning and outcome is moderated by attachment anxiety condition. (e.g. high versus low anxious attachment)

\section{Hypotheses}

Five research hypotheses are listed below to test the research objectives.

H1: The reliability between raters coding the DMRS defensive functioning score will meet standards of good inter-rater reliability.

H2: Overall defensive functioning scores will be negatively correlated with pre-treatment scores of attachment anxiety and attachment avoidance, depression, interpersonal problems and positively correlated with self-esteem. 
H3: Overall defensive functioning scores will show significant improvement over the course of the 16-week group psychodynamic interpersonal psychotherapy. (i.e., across sessions 3, 8, and 16).

H4: Increases in overall defensive functioning scores during treatment will be associated with decreases in episodes of binge eating, depressive symptoms, and interpersonal problems, as well as an increase in self esteem from pre-treatment to one year follow up. H5: The relationship between change in overall defensive functioning scores and symptom change will be moderated by attachment anxiety condition. Specifically, the relationship between defensive functioning scores and treatment outcome will be stronger in the high attachment anxiety condition compared to the low attachment anxiety condition.

\section{Method}

\section{Participants}

This study was based on archival data. Adult participants were recruited through advertisement in local newspapers and through referrals from an eating disorder treatment center. Participants met the diagnostic criteria for BED based on the DSM-IVTR (APA, 2000), had a Body Mass Index (BMI) greater than 27 (overweight), were 18 years or older, were women, and were able to read and speak in English. Prospective participants were screened and excluded if they reported: current or past incidences of inappropriate compensatory behaviour (such as laxative use or vomiting), alcohol or drug abuse in the past six months, use of any medications that might affect weight over the course of treatment, a diagnosis or bipolar or any psychotic disorder, current or future plans to become pregnant during treatment, and plans to enrol in a weight loss program. 
Eligible participants were then assigned to one of two therapy conditions based on scores obtained from a measure of attachment completed before the 16-week therapy began. One condition was assigned based on high scores on the Need for Approval subscale of the ASQ $(n=50)$ and the other based on low Need for Approval scores $(n=52)$ based on Tasca et al. (2006) study, which will be discussed below.

Six female therapists (two psychiatrists, three psychologists, and one masters level advanced practice nurse) conducted the 16-week group therapy sessions. Each therapist was assigned two therapy groups; one high in attachment anxiety and one low in attachment anxiety. Therapists were blind to attachment condition. Each therapist had at least three years experience providing group psychotherapy. Training and oversight included a two-day GPIP training workshop, as well as weekly supervision with a senior psychologist experienced in GPIP, eating disorders and group therapy to ensure adherence to the treatment manual. Treatment adherence was previously assessed in another study using the Tape Rating Instrument for Psychotherapy of Eating Disorders (TRIPED; Olmsted, Isaacs, Bemis, \& Garner, 1988). Scores of three or above are within the acceptable limits of adherence. For this study, mean TRIPED score was $3.88(S D=$ 0.55) indicating adequate treatment adherence (Tasca et al., 2013).

\section{Measures}

Attachment Styles Questionnaire (ASQ; Feeney, Nollar, \&Hanrahan, 1994). The ASQ is a widely used self-report measure to assess adult attachment (see Appendix C). It consists of 40 items rated on a six-point scale ( $1=$ totally disagree, and $6=$ totally agree) and comprising five dimensions, which include two scales measuring attachment anxiety (Need for Approval, and Preoccupied with Relationships), two measuring 
attachment avoidance (Discomfort with Closeness, and Relationships as Secondary), and one scale measuring attachment security (Confidence in Relationships). Higher scores indicate greater attachment security or greater insecurity for each dimension. The Need for Approval scale of the ASQ loads highly onto the attachment anxiety factor (Brennan, Clark, \& Shaver, 1998). The range of Cronbach's alpha at pre, post, 6 months, and 12 months post-treatment for each subscale was: .80 to .84 for Confidence in Relationships, .80 to 85 for Discomfort with Closeness, .77 to .81 for Need for Approval, .71 to .80 for Preoccupied with Relationships, .66 to .78 for Relationships as Secondary. Mean interitem correlation was also used to assess internal consistency between scale items. The number of scale items can influence the value of Cronbach's alpha, in which scales with greater items can produce higher coefficient alpha values. For this reason, I also report mean inter-item correlations, which are not influenced by the number of scale items (Clark \& Watson, 1995). Inter-item correlations between .15 and .50 are considered adequate (Clark \& Watson, 1995). The mean inter item correlation for each scale was between .25 and .41 , indicating adequate internal consistency.

Beck Depression Inventory II (BDI; Beck, Steer, \& Brown, 1996). The BDI-II is a widely used 21 item self-report measure of depressive symptoms and attitudes. Total scores on the BDI were used in the analysis, and is referred to as depressive symptoms in the results and discussion section. Each question is rated on a 4-point Likert scale ranging from 0 to 3, with higher scores indicating greater depressive symptoms (see Appendix D). Cronbach alpha for this study ranged from .92 at pre-treatment and post-treatment, .90 at 6 months post-treatment, and .92 at 12 months post-treatment. Mean inter item 
correlation was used to assess internal consistency, which for this study was .35 , indicating adequate internal consistency.

Eating Disorder Examination (EDE; Fairburn \& Cooper, 1993). The EDE is a semi-structured investigator interview developed by Fairburn and Cooper (1987) was used to assess days binged in the past 28 days. The original treatment study used the twelfth edition of the EDE, which included a module on Binge Eating Disorder based on the DSM-IV criteria (Fairburn, 2008; Fairburn \& Cooper, 1993). Participants in the study were assessed with the EDE at pre-treatment, in-person at 6-month follow up, and over the phone at 12-month follow-up. The number of days binged in the past 28 days was the primary interest and is referred to as Binge Frequency in the results and discussion section. Inter rater reliability of days binged was assessed by having a blind independent rater reassess $10 \%$ of the recorded interviews. The two-way random effects model intraclass correlation was .98 , which indicates excellent agreement between raters.

Defence Mechanism Rating Scale (DMRS) (Perry, 1990). The DMRS is an observer rated measure of defence mechanisms comprised of 27 defences organized into seven levels, which are weighted from low adaptive to high adaptive (see Table 1). Defences are coded then multiplied by the level weight, then the weighted average of all defences is calculated (see Appendix 1). This score is the ODF, which is a measure of the relative adaptiveness of defensive functioning and ranges from 1 to 7 , in which higher scores indicate higher adaptive functioning and low scores indicate less adaptive functioning (Perry \&Høglend, 1998). The mean ODF score for patients diagnosed with depression was $4.41(S D=.82)$ (Perry \&Høglend, 1998). The mean ODF score obtained from a sample consisting predominantly of patients with mood disorders seeking 
psychotherapy services was $4.97(S D=.70)$ (Hilsenroth, Callahan, \&Eudell, 2003).

Finally, in a sample of inpatients and outpatients seeking psychological treatment, the mean ODF score was $4.48(S D=.93)$. ODF scores for patients undergoing psychodynamic therapy for personality disorders rose from $4.41(S D=.65)$ at pretreatment to $5.26(S D=1.02)$ measured at session 36 (Johansen et al., 2011).

The DMRS is one of the most widely used observer-rated measures and is a versatile tool for measuring clinical interviews and life vignette data (Drapeau et al., 2011). Reliability scores for the DMRS differ based on assessment of individual defences compared to defence levels. Reliability for individual defences has been shown to be low (.36), while reliability for defence levels is higher (.53) (Presniak et al., 2010) though still below the threshold of good reliability (ICC > .61) (Landis \& Koch, 1977). Reliability scores for individual (.57) and defence level scores (.74) improve when raters reach a consensus (which will be discussed below), that is, when raters code transcripts together and reach agreement on defence coding.

\section{Inventory of Interpersonal Problems (IIP; Horowitz, Rosenberg, Baer,}

Ureno, \&Villasenor, 1988). The IIP is 64-item self-report measure of interpersonal distress. For this study, total scores on the IIP were used as an indicator of current distress (see appendix E) and is referred to as interpersonal problems in the results and discussion section. The coefficient alphas for the total score were .85 when measured at pretreatment, post-treatment, 6 months post-treatment, and 12 months post-treatment. Mean inter item correlation was .42 indicating good reliability (Watson \& Clark, 1995).

Rosenberg Self-Esteem Scale (RSES; Rosenberg, 1965). The Rosenberg SelfEsteem Scale is a 10 item self-report measure (see Appendix F). Mean scores on the 
RSES were used in the analysis, referred to as self-esteem in the results and discussion. Items are rated on a four-point scale ranging from Strongly Disagree to Strongly Agree. Cronbach's alpha for this study was .92 at pre-treatment, post-treatment, 6 months posttreatment, and 12 months post-treatment. Mean inter item correlation was .37 for this study.

\section{Procedure}

Prospective participants were initially screened for the presence of BED by a research coordinator. Eligible participants then completed a pre-treatment questionnaire package that included measures of attachment, depression, self-esteem, interpersonal difficulty, and an assessment of the number of days binged in the past 28 days with the EDE. Participants also met with an experienced therapist for a pre-group preparation session.

Participants were then assigned into one of two conditions based on their pretreatment score on the Need for Approval sub-scale of the ASQ. For this study, the cutoff score for the Need for Approval scale was 3.59. Scores equal-to or greater-than 3.59 were placed in the high attachment anxiety condition, while scores lower than 3.59 were placed in the low attachment anxiety condition. The cut-off score was selected based on Tasca et al. (2006) study that found the regression line for the interaction of Need for Approval and treatment type (CBT or GPIP) intersected at the value 3.59 (Tasca et al., 2006).

Each group in each condition received 16 sessions of GPIP, and each session was video recorded to ensure adherence to the treatment manual, and for future research. Once the 16 sessions were complete, participants were given a post-treatment 
questionnaire package. A follow up package was given to participants again 6-months post-treatment and 12-months post-treatment to assess outcomes.

Three sessions from each of the 12 therapy groups were transcribed. Sessions 3, 8, and 16 were selected to provide a consistent view of defensive functioning throughout treatment. Thirty-six sessions, each lasting approximately 90 minutes, were coded on the DMRS by myself and a group of reliable raters. Each rater coded approximately two sets of three transcripts and consensus coded two additional transcripts that were already coded by another rater to improve reliability and reduce rater drift. Raters coded transcripts and video recordings for defensive functioning for sessions 3, 8, and 16 of two separate therapy groups. We de-identified therapy sessions to mask the session number. However, it was still possible for coders to determine what therapy session was being coded either through group member's discussion of what session it was, or by the therapist announcing the session number at the start of the group. Raters were blind to attachment anxiety condition during the coding process to avoid potential rater bias.

\section{DMRS Coding}

Each rater received extensive training on use of the DMRS for coding defensive functioning. Training occurred over the course of several months by an experienced psychologist trained by Dr. Chris Perry in use of the DMRS. Dr. Perry also provided occasional consultation on coding issues that arose within the group context. Only those raters who attained an adequate level of reliability, based on the standards of good reliability identified by Dr. Perry (ICC > .70), coded transcripts. In addition to myself, the raters included 2 psychologists, 1 resident psychiatrist, 1 doctoral student in psychology, and 1 undergraduate student in psychology. Raters coded defences by watching the video 
of the group therapy session and coding any identified defence mechanisms on a paper transcript of the therapy session. Each set of sessions were randomized and de-identified so that raters were unaware of which session they were coding. Raters were also blind to the attachment anxiety condition of each group, that is, which group was high or low on attachment anxiety condition. The entire 90-minute GPIP session was coded and each rater completed an ODF scoring sheet (see Appendix A) for each participant had a codeable defence mechanism in a session.

Raters also engaged in consensus coding for a proportion of therapy sessions. Twelve of the 36 therapy sessions were consensus coded to assess reliability and to reduce rater drift. Raters met with their coding partner, who was another trained investigator, and they reached consensus on any disagreement on coded defences identified in each of the two sessions. In addition, meetings were held every two to three weeks with a senior clinician trained in using the DMRS, and each rating pair presented issues with coding or any defences on which they could not reach consensus.

\section{Data Analysis Plan}

Prior to analysis, the data were screened for the presence of univariate outliers. Scores that fell outside of 3.29 standard deviations from the mean were considered outliers and were adjusted to within 3.29 standard deviations from the mean. Multivariate outliers were examined by testing the Mahalanobis distance, the Leverage, Studentized Residual, and DFBeta/DFfit scores for each variable. Skewness and Kurtosis were assessed and tested by dividing the skewness or kurtosis value by its associated standard error. Values greater than 3.29 were considered skewed or kurtotic in which case 
transformations were considered. To assess multicollinearity, tolerance scores were obtained and examined.

Due to the nature of group therapy in which not all participants speak at the same frequency during a session, there was a wide range in the number of defences coded for each participant in a given session (Table 2). Some participants talked more during the session, while others talked little or not at all. The number of defences coded by raters were used to filter cases when testing for reliability. The reliability scores obtained when cases were filtered by the number of defences coded per person helped inform what the minimum number of coded defences would be for the analyses. That is the minimum number of coded defenses per person in order to include their data in the analyses was determined by the point at which an adequate inter-rater reliability was achieved. This preserved adequate reliability between raters while maintaining a sufficient sample size.

Table 2

Mean, Standard Deviation, and Range of the Number of Coded Defences by Therapy Session

\begin{tabular}{lccccc}
\hline & & & & \multicolumn{2}{c}{ Range } \\
\cline { 5 - 6 } & $N$ & $M$ & $S D$ & Min & Max \\
\hline Session 3 & 44 & 15.73 & 11.78 & 5 & 59 \\
Session 8 & 49 & 17.16 & 13.21 & 5 & 75 \\
Session 16 & 42 & 11.19 & 6.10 & 5 & 34 \\
\hline
\end{tabular}

The first step in the analysis was to assess the reliability between coding pairs using the DMRS. A one-way random effects intra-class correlation coefficient (ICC; 1, 1) with absolute agreement was used to calculate inter-rater reliability of ODF scores obtained from paired raters coding the same session. The ICC is a recommended measure of reliability for quantitative data because it accounts for chance agreement 
(Bartko\&Carpenter, 1976) and is often used to test reliability for the DMRS (Hilsenroth et al., 2003; Johansen et al., 2011; Perry et al., 2009; Presniak et al., 2009). The one-way random effects model is appropriate when each session is rated by a different set of judges who were randomly selected from a larger population of judges (Shrout\& Fleiss, 1977). In the current study, rating pairs also engaged in consensus coding to reduce rater drift. That is, 12 transcripts that were coded by a pair of raters were compared and differences in coding decisions were resolved by consensus between the raters. Previous research used consensus coding to boost inter-rater reliability by comparing consensus scores rather than independent rater scores (Johansen et al., 2011; Perry et al., 2009). However, this likely inflated true inter-rater reliability, and so consensus codes were not included in estimating reliability in the current study. To test the first hypothesis, an ICC was calculated for independent coding of pairs of raters of 12 of the 36 transcripts. ICC values greater than .60 are considered indicators of "good reliability" (Landis \& Koch, 1977). To test the second hypothesis, a Pearson product-moment correlation was used to test the associations between session three ODF scores and pre-treatment scores on the BDI, IIP, RSES and Binge Frequency. A post-hoc correlation analysis was also conducted to test the associations between session 16 ODF scores and post-treatment scores on the BDI, IIP, RSES and Binge Frequency.

Multi-Level Modeling. Multi-Level Modeling (MLM) was used to test the remaining hypotheses. All MLM analyses were conducted with HLM 7 software with full maximum likelihood estimation. MLM has several advantages over ordinary least squares (OLS) regression and is ideally suited for testing nested data (i.e., repeated measurements within individuals, or individuals within groups; Raudenbush\&Bryk, 
2002). I began by testing the assumptions for a MLM by screening for the presence of outliers (including slopes and intercepts), linearity, normality, homoscedasticity, homogeneity and independence of variance, and independence of error terms between levels of the model.

MLM is robust when there are missing values. Data can be analyzed without listwise deletion or data imputation if the data are missing at random (MAR). Missing at random refers to the missingness pattern being independent of levels of the outcome variable, although the pattern can still be dependent on other variables in the analysis (Gallop \&Tasca, 2009). A pattern mixture model was used to assess if the data was MAR, including patterns for any missing values, and treatment completer status. Missing data patterns that were significantly associated with dependent variables were entered at Level-2 of the MLM as control variables.

Longitudinal data and growth curve modeling can be viewed as multi-level; that is, repeated measurement data at Level-1 are nested within-person at Level-2, and persons are nested within groups at Level-3. Within-person parameters occupy Level-1 of the model, with individual time points for a participant. The between-person parameters occupy Level-2 and are comprised of the slope and intercept values generated from the within-person Level-1 data. Between-group parameters occupy Level-3 and consist of nested Level-1 and Level-2 data.

In the current study, individual participants were nested within 12 therapy groups. Group treatment data may be dependent within groups, which can inflate Type I error rate by estimating inaccurate standard errors (Tasca et al., 2009). To assess dependence, a 3-Level conditional model with attachment anxiety condition was specified as was a 3- 
Level unconditional model with attachment anxiety condition not specified. From these models the Level-3 between-group slope variance component $\left(\tau_{\square \square)}\right.$, as well as the unconditional Level-2 slope variance component $\left(\tau_{\square \text { ij }}\right)$ were obtained. The intra class correlation coefficient to assess data dependence, $\rho$, was calculated using the equation, $\rho$ $=\tau_{10 \mathrm{j}}$ (conditional) $/\left(\tau_{10 \mathrm{j}}\right.$ (unconditional $\left.)+\tau_{1 \mathrm{ij}}\right)$ (Tasca, et al., 2009). A $\rho$ value less than .05 indicates less than $5 \%$ of the slope variance is between groups, indicating that dependence is likely ignorable and a 2-level model can be used (Kenny, Kashy, \& Bolger, 1998). If $\rho$ is greater than .05, the data dependence is not ignorable (Kenny et al., 1998), in which case one can use a 2-Level MLM, but Type 1 error rate must be adjusted (Tasca, et al., 2009).

To assess improved model fit, effect sizes were evaluated with the pseudo- $R^{2}$, which is the percent change in variance components due to the addition of a predictor parameter (Tasca\& Gallop, 2009). Pseudo- $R^{2}$ is calculated using the formula $\left(\sigma_{\mathrm{u}}^{2}-\sigma_{\mathrm{t}}^{2}\right) /$ $\sigma_{\mathrm{u}}^{2}$ ), in which $\sigma_{\mathrm{u}}^{2}$ is the variance component associated with the previous model, and $\sigma_{\mathrm{t}}^{2}$ is the variance component associated with the subsequent nested model that includes the parameter of interest (Raudenbush\&Bryk, 2002). In all models, baseline values were controlled at Level-2 to account for the effect of individuals' starting values on their slope parameters. This was done by grand mean centering pre-treatment outcome scores or ODF session 3 scores, and including them as control variables at level 2.

The MLM equations are included in appendix G. To test hypothesis 3, a growth curve analysis of the data was used to examine change in ODF. A linear growth model is useful for examining trends in data when the amount of observations per person is low (e.g. three or four observations; Raudenbush\&Bryk, 2002). I used a 2-Level 
unconditional linear growth model to test the third hypothesis. ODF scores were the outcome variable at Level 1 with linear time entered as the Level-1 predictor. Session 3 ODF scores were grand mean centered at Level-2 to control for baseline scores. As will be indicated in the Results section, completer status was entered at Level-2 as a control variable (See Appendix G).

To test hypothesis 4, ODF OLS regression slope values for each individual were taken from the 2-Level growth model used to test hypothesis 3 . These slope values indicate rates of change in ODF for each individual. ODF slope values were then entered as Level-2 predictor variables for each outcome (depressive symptoms, interpersonal problems, self-esteem, and binge frequency; see Appendix G) to test the association between change in outcome from pre-treatment to 12 months follow up with change in ODF during group therapy. Based on previous research using the same data, the outcome variables' growth was modeled as a log-linear trend characterized by a sharp negative curvilinear decrease in scores on depression, interpersonal problems and binge frequency from pre to post-treatment, followed by a more gradual decline in symptoms over the follow up periods. Change in self-esteem was expected to follow the same pattern, but positive over time.

To test hypothesis 5, ODF slope values, baseline pre-treatment scores, and attachment anxiety condition were entered at Level-2 of each model assessing change in treatment outcome (depressive symptoms, interpersonal problems, self-esteem, binge frequency). An attachment anxiety condition x ODF slope interaction term was created and entered at Level-2 of the model to test if the association between change in defensive functioning and change in treatment outcome was moderated by high or low attachment 
anxiety (Appendix G; Model 4). Follow up models were specified to assess any moderating effects indicated by significant interactions. Separate 2-Level models tested whether change in defensive functioning scores were associated with change in the outcomes for participants high in the attachment anxiety or low attachment anxiety condition.

\section{Results}

\section{DataScreening}

Two scores were identified as outliers, one on the post-BDI measure and the other on the 6-month post-BDI measure. Both scores were adjusted to within 3.29 SD from the mean, while remaining the highest score in the distribution. No multivariate outliers were identified and there was no evidence of multicollinearity. The majority of the data was normally distributed, however the 6-month and 12-month BDI scores, as well as the 6month and 12-month binge frequency variables were significantly positively skewed. Transforming the variables only at the time points in which the skewness occurred would change their metric relative to other time points and make the MLM results uninterpretable. Therefore the skewed 6-month, 12-month BDI and binge frequency variables were not transformed.

\section{Sample Characteristics}

Prospective participants were screened for inclusion into the study. One hundred and two female participants with BED began treatment. The participants were assigned to a high attachment anxiety condition $(n=52)$ and a low attachment anxiety condition $(n=$ 50). Seventy-seven participants had ODF scores based on five or more defence mechanisms in at least one therapy session. Analyses conducted for hypothesis 3, 4, and 
5 were completed with $N=77$. Lower sample sizes in any analysis were the result of missing data at Level-1 of the MLM. Table 3 provides demographic information of the sample used in the analyses.

Table 3

Sample Characteristics

\begin{tabular}{lrcc}
\hline \multicolumn{1}{l}{ Language } & & $n$ & Percent \\
& English & 64 & 85.3 \\
& French & 5 & 6.7 \\
& Bilingual & 6 & 8 \\
\hline Marital Status & Single & 21 & 27.6 \\
& 37 & 48.7 \\
Married/Common Law & 37.1 \\
Separated/Divorced & 16 & 21.1 \\
& Widowed & 3 & 2.6 \\
\hline
\end{tabular}

Living Arrangement

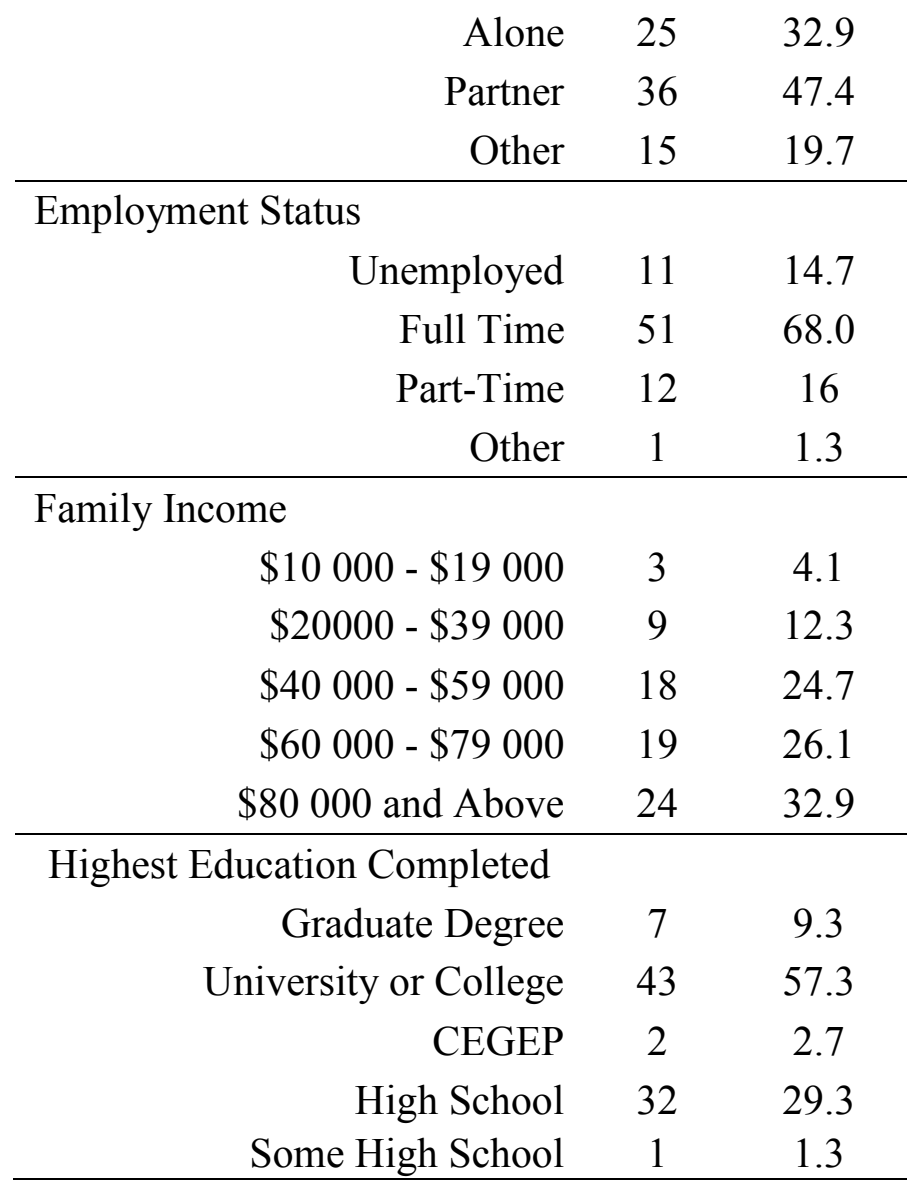


Note. Frequency values based on $N=77$. Some values are lower due to missing data

To test the first hypothesis, a one-way random effects model $\operatorname{ICC}(1,1)$ was used to test for good reliability among raters. Reliability was tested using only the ODF scores coded by individual raters and not the consensus coded values. In total, 24 therapy sessions were consensus coded by rating pairs. ODF scores for 70 participants were available for the reliability analysis. Overall reliability between rating pairs when coding ODF scores was .59, which is below the standard of "good" reliability (Landis \& Koch, 1977). The ICC $(1,1)$ value increased to .70 when reliability was calculated using ODF scores based on 5 or more coded defences per person $(\mathrm{N}=44)$, thus indicating "good" agreement between raters (Landis \& Koch, 1977). Hence, for subsequent analyses, we retained only participant data based on 5 or more ODF scores.

ICC values for each defence level of the DMRS for the 44 individuals with greater than 5 coded defenses are presented in Table 4. Consistent with previous research, reliability was lower for some of the defence level scores relative to overall ODF scores (Perry, 2009; Presniak et al., 2010). Nevertheless, defence levels provide useful descriptive information about which specific defences were coded for each participant. Major Image Distorting fell within the range of "excellent" reliability (ICC > .80; Landis \& Koch, 1977). Obsessional, Minor Image Distorting, and Action level defences were all within the range of "good" reliability (ICC > .60; Landis \& Koch, 1977). Disavowal and High Adaptive level defences were on the threshold for "good" reliability. Other Neurotic level defences were below the threshold for "good" reliability. 
Table 4

Defence level ICC Values and Total Number of

Defences Coded Between Rating Pairs.

\begin{tabular}{|c|c|c|c|}
\hline Level & & $\begin{array}{l}\text { Frequency of } \\
\text { Defences }\end{array}$ & $\mathrm{ICC}$ \\
\hline 7 & High Adaptive & 230 & .59 \\
\hline 6 & Obsessional & 576 & .79 \\
\hline 5 & Other Neurotic & 342 & .36 \\
\hline 4 & Minor Image Distorting & 133 & .65 \\
\hline 3 & Disavowal & 309 & .60 \\
\hline & Fantasy & 0 & -- \\
\hline 2 & Major Image Distorting & 47 & .82 \\
\hline 1 & Action & 82 & .73 \\
\hline \multicolumn{4}{|c|}{$\begin{array}{l}\text { Note. } N=44 \text { individuals for the reliability analysis } \\
\text { who had } 5 \text { or more coded defences. ICC = Intraclass } \\
\text { Correlation Coefficient one-way random effects } \\
\text { model. Frequency of Defences represent the total } \\
\text { number of defences coded by rating pairs for each } \\
\text { level. No instance of Fantasy was coded in this } \\
\text { sample and so ICC was not calculated. }\end{array}$} \\
\hline
\end{tabular}

Hypothesis two predicated that session 3 ODF scores would be correlated with pre-treatment depressive symptoms, interpersonal problems, self-esteem, binge frequency, and attachment styles subscales. Table 5 shows means and standard deviations of the attachment scales for pre and post-treatment separated by attachment anxiety condition. Contrary to the second hypothesis, there was no significant correlation between any of the pre-treatment outcome measures or attachment scales with session 3 ODF scores (Tables 6 and 7). As a post-hoc analysis, we tested the association between session 16 ODF scores and post-treatment outcome scores, as well as the five attachment styles subscales. The results indicate that ODF scores from session 16 were moderately and significantly negatively correlated with post-treatment depressive symptom, and binge frequency (Table 8) and negatively correlated with Confidence in Relationships, which measures attachment security (Table 9). 
Table 5

Mean and Standard Deviations for Attachment Style Questionnaire Subscales at Pre-treatment and Posttreatment by Attachment Anxiety Condition

\begin{tabular}{|c|c|c|c|c|c|c|c|c|c|c|}
\hline & \multicolumn{4}{|c|}{ Pre-treatment } & \multicolumn{6}{|c|}{ Post-treatment } \\
\hline & \multicolumn{2}{|c|}{ High } & \multicolumn{2}{|c|}{ Low } & \multirow[b]{2}{*}{$t$} & \multicolumn{2}{|c|}{ High } & \multicolumn{2}{|c|}{ Low } & \multirow[b]{2}{*}{$t$} \\
\hline & $M$ & $S D$ & $M$ & $S D$ & & $M$ & $S D$ & $M$ & $S D$ & \\
\hline Confidence in Relationships & 3.29 & .73 & 4.26 & .71 & $5.96 *$ & 3.82 & .58 & 4.35 & .86 & $2.99 *$ \\
\hline Discomfort with Closeness & 4.56 & .73 & 3.60 & .73 & $-5.74 *$ & 4.02 & .65 & 3.47 & .89 & $-2.84 *$ \\
\hline Need for Approval & 4.75 & .45 & 3.28 & .55 & $-12.79 *$ & 3.91 & .74 & 3.05 & .71 & $-4.99 *$ \\
\hline $\begin{array}{l}\text { Preoccupation with } \\
\text { Relationships }\end{array}$ & 4.31 & .62 & 3.56 & .82 & $-4.55^{*}$ & 3.75 & .51 & 3.28 & .86 & $-2.71 *$ \\
\hline Relationships as Secondary & 2.98 & .57 & 2.34 & .61 & $-3.95^{*}$ & 2.45 & .72 & 2.22 & .58 & -1.51 \\
\hline$N$ & 37 & & 40 & & & 32 & & 38 & & \\
\hline
\end{tabular}

$$
* p<.01
$$

Note. High refers to the High Attachment Anxiety Condition. Low Refers to the Low Attachment Anxiety Condition. Degrees of freedom for the pre-treatment t-test was 75. Degrees of freedom for the post-treatment t-test was 68. Differences in sample size from pre-treatment to post-treatment due to missing data and noncompletion of treatment. 
Table 6

Correlations Between Session Three ODF Scores and Pre-treatment Measures of Depression, Interpersonal Problems, Self Esteem, and Binge Frequency

\begin{tabular}{lccccc}
\hline Pre-treatment & 1 & 2 & 3 & 4 & 5 \\
\hline 1 Overall Defensive Functioning & - & -.02 & .02 & -.01 & -.12 \\
2 Pre Depressive Symptoms & - & - & $.43^{* *}$ & $-.62 * *$ & $.21 *$ \\
3 Pre Interpersonal Problems & - & - & - & $-.53^{* *}$ & .14 \\
4 Pre Self-Esteem & - & - & - & - & -.01 \\
5 Pre Binge Frequency past 28 days & - & - & - & - & - \\
\hline
\end{tabular}

$* p<.01$ (two tailed) $* * p<.05$ (two tailed)

Note. ODF= Overall Defensive Functioning. $N=77$ for Depressive Symptoms, Interpersonal Problems, Self-Esteem, and Binge Frequency. $N=44$ for session 3 overall defensive functioning. Correlation conducted with imputed data, but no significant differences were found. Original results are presented.

Table 7

Correlations Between Session 3 ODF Scores with Pre-treatment Subscales from the Attachment Styles Questionnaire

\begin{tabular}{llrrrrc}
\hline Pre-treatment & 1 & 2 & 3 & 4 & 5 & 6 \\
\hline 1 Overall Defensive Functioning & - & .06 & -.13 & .08 & -.09 & -.02 \\
2 Confidence in Relationships & - & - & $-.74^{* *}$ & $-.69^{* *}$ & $-.48^{* *}$ & $-.48^{* *}$ \\
3 Discomfort with Closeness & - & - & - & $.62^{* *}$ & $.35^{* *}$ & $.56^{* *}$ \\
4 Need for Approval & - & - & - & - & $.67^{* *}$ & $.47^{* *}$ \\
5 Preoccupation with Relationships & - & - & - & - & - & .21 \\
6 Relationships as Secondary & - & - & - & - & - & - \\
\hline
\end{tabular}
$* p<.05$ (two tailed) $* * p<.01$ (two tailed)

Note. ODF $=$ Overall Defensive Functioning. $N=77$ for ASQ Subscales. $N=44$ for Session 3 ODF scores. Correlation conducted with imputed data, but no significant differences were found. Original results are presented. 
Table 8

Correlations Between Session 16 ODF Scores with Post-treatment Measures of Depression, Interpersonal Problems, Self Esteem, and Binge Episodes

\begin{tabular}{llcccc}
\hline Post-treatment & 1 & 2 & 3 & 4 & 5 \\
\hline 1 Overall Defensive Functioning & - & $-.35^{*}$ & .16 & -.04 & $-.39^{*}$ \\
2 Depressive Symptoms & - & - & $.46^{* *}$ & $-.57^{* *}$ & $-.27^{*}$ \\
3 Interpersonal Problems & - & - & - & -.50 & -.07 \\
4 Self-Esteem & - & - & - & - & -.03 \\
5 Binge Frequency past 28 days & - & - & - & - & - \\
\hline$* p<.05$ (two tailed) $* * p<.01$ (two tailed) & & & &
\end{tabular}

$* p<.05$ (two tailed) $* * p<.01$ (two tailed)

Note. ODF $=$ Overall Defensive Functioning. $N=42$ for Session 16 ODF scores, and $N=70$ for Depressive Symptoms, Interpersonal Problems, Self

Esteem, Binge Frequency due to non-completers and missing data. Correlation conducted with imputed data, but no significant differences were found.

Original results are presented.

Table 9

Correlations Between Session 16 ODF Scores with Post-treatment Subscales from the Attachment Styles Questionnaire

\begin{tabular}{llccccc}
\hline Post-treatment & 1 & 2 & 3 & 4 & 5 & 6 \\
\hline 1 Overall Defensive Functioning & - & $-.34^{*}$ & .18 & .22 & .22 & .09 \\
2 Confidence in Relationships & - & - & $-.71^{* *}$ & $-.58^{* *}$ & $-.41^{* *}$ & $-.48^{* *}$ \\
3 Discomfort with Closeness & - & - & - & $.51^{* *}$ & $.29^{*}$ & $.41^{* *}$ \\
4 Need for Approval & - & - & - & - & $.51^{* *}$ & $.50^{* *}$ \\
5 Preoccupation with Relationships & - & - & - & - & - & .21 \\
6 Relationships as Secondary & - & - & - & - & - & - \\
\hline
\end{tabular}

$* p<.05$ (two tailed) $* * p<.01$ (two tailed)

Note. ODF $=$ Overall Defensive Functioning. $N=42$ for session 16 ODF scores, and $N$ $=69$ due to non-completers and missing data. Correlation conducted with imputed data, but no significant differences were found. Original results are presented.

\section{Multi-level Modeling}

Preliminary analyses.Approximately $35-45 \%$ of the 77 participants in the analysis had missing ODF scores in at least one of the three group therapy sessions (42.9\% session $3 ; 32.5 \%$ session $8 ; 45.5 \%$ session 16$)$. I conducted two pattern mixture 
models to assess if patterns of missing data were associated with ODF slope parameters (Gallop \&Tasca, 2009). The patterns tested were: (1) missing any data, and (2) missing data caused by treatment non-completion. The slope parameter for the missing data pattern caused by treatment non-completion was significant $\left(\beta_{11}=-.93, S E=.20, t_{(74)}=\right.$ 4.66, $p<.001)$, indicating that ODF change was related to treatment non-completion so that treatment completers showed greater positive ODF rate of change. Therefore a predictor representing the completer status pattern was included as a level-2 covariate when testing MLM of ODF scores.

As indicated previously, baseline scores of individuals were controlled when estimating slopes. In the case of missing baseline data, session 3 ODF scores were imputed based on full maximum likelihood estimates of the intercepts from the unconditional linear model. Imputation in this manner is effective and desirable in MLM because it provides robust results even if the data are not MAR (Raudenbush\&Bryk, 2002). Subsequent analyses used the imputed session 3 values when controlling for ODF baseline.

The percent missing data among the predictor variables was low (below 5\%) for pre-treatment and post-treatment scores, but increased to $29.9 \%$ for BDI, RSES, and Binge Frequency scores and 32.5\% for IIP scores at 6 months post-treatment. Missing values from the 12-month post-treatment follow up ranged from 51\% (BDI, Binge Frequency) to $52 \%$ (IIP, RSES). According to the missing data pattern mixture model analyses, there was no pattern that was significantly associated with the slope parameters. Hence the data were MAR and MLM were run without modification. 
The ODF data were tested for dependence due to individuals nested within groups. This analysis produced an intra class correlation coefficient, $\rho=.07$, indicating some dependence in the grouped ODF data. Hence, for two-level MLM of ODF scores I used an adjusted Type I error rate of .03 based on tables provided by Kenny et al. (1998). An independent sample t-test demonstrated that mean overall ODF scores in the high attachment anxiety condition $(M=5.33, S D=.54)$ differed significantly from the low attachment anxiety condition and the effect size was medium $(M=4.90, S D=.77 ; t(75)=$ $-2.86, p<.01, d=.65)$. Table 10 displays the mean sample ODF scores by attachment anxiety condition for sessions 3,8 , and 16 . Follow up independent samples $t$-tests were used to test differences in ODF scores between attachment anxiety conditions by group therapy session. Session 8 scores were significantly different between attachment conditions, whereas session 3 and 16 ODF scores were not significantly different. Table 11 displays the means and standard deviation for each variable tested from pre-treatment to 12 months post-treatment.

Table 10 Mean and SD Values for ODF Scores at Sessions 3, 8, and 16

\begin{tabular}{ccccccccc}
\hline & \multicolumn{2}{c}{$\begin{array}{c}\text { Aow Attachment } \\
\text { Anxiety }\end{array}$} & \multicolumn{2}{c}{$\begin{array}{c}\text { High Attachment } \\
\text { Anxiety }\end{array}$} & \multicolumn{3}{c}{ Total Sample } \\
\hline Session & $M(S D)$ & $N$ & $M(S D)$ & $N$ & $t$-test & $d$ & $M(S D)$ & $N$ \\
\hline 3 & $4.85(.71)$ & 19 & $5.03(.67)$ & 25 & -0.87 & .26 & $4.95(.67)$ & 44 \\
8 & $4.77(.88)$ & 25 & $5.30(.53)$ & 26 & $-2.63 *$ & .73 & $5.04(.78)$ & 51 \\
16 & $5.30(.73)$ & 18 & $5.56(.80)$ & 24 & -1.46 & .34 & $5.50(.78)$ & 42 \\
\hline
\end{tabular}

$* p=.01$

Note. Total sample size smaller than $N=77$ due to missing data. Results did not differ when tested with imputed values. ODF $=$ Overall Defensive Functioning. 
Table 11

Mean and SD for Treatment Outcome Variables from Pre-treatment to 12-Months Post-treatment

\begin{tabular}{|c|c|c|c|c|c|c|c|c|c|}
\hline & & \multicolumn{2}{|c|}{ Pre-treatment } & \multicolumn{2}{|c|}{ Post-treatment } & \multicolumn{2}{|c|}{ Six Months } & \multicolumn{2}{|c|}{12 Months } \\
\hline & & $M$ & $S D$ & $M$ & $S D$ & $M$ & $S D$ & $M$ & $S D$ \\
\hline \multirow[t]{2}{*}{ Binge Frequency Past 28 Days } & & 15.44 & 5.67 & 7.34 & 7.60 & 6.55 & 8.03 & 5.68 & 6.78 \\
\hline & $N$ & 77 & & 71 & & 57 & & 39 & \\
\hline \multirow[t]{2}{*}{ Beck Depression Inventory } & & 20.34 & 10.93 & 12.28 & 10.41 & 12.28 & 10.41 & 13.17 & 10.71 \\
\hline & $N$ & 76 & & 69 & & 54 & & 39 & \\
\hline \multirow[t]{2}{*}{ Interpersonal Problems } & & 93.18 & 36.64 & 83.44 & 33.31 & 77.69 & 33.3 & 73.15 & 35.01 \\
\hline & $N$ & 75 & & 70 & & 52 & & 40 & \\
\hline \multirow[t]{2}{*}{ Rosenberg Self-Esteem } & & 2.63 & .65 & 2.93 & .57 & 3.05 & .61 & 3.02 & .59 \\
\hline & $N$ & 77 & & 70 & & 54 & & 40 & \\
\hline
\end{tabular}

Note. Sample sizes are less than 77 in some cases due to missing data. $S D=$ Standard Deviation. 
Main Analyses. The third hypothesis stated that there would be a positive change in ODF scores across group treatment sessions. The results of the 2-Level growth model indicated a significant positive linear change in ODF from session 3 to session 16 and effects were large (Table 12). The growth trend of the outcome variables for the sample of participants in this study was also tested. Depressive symptoms, interpersonal problems, self esteem, and binge frequency improved precipitously from pre-treatment to post-treatment and improved to a lesser degree from post-treatment to 12 months posttreatment. Self-esteem scores significantly increased greatly from pre-treatment to posttreatment, and less so from post-treatment to 12 months post-treatment. Effect sizes for growth in the outcomes were all large (Table 12). These significant loglinear growth patterns of the treatment outcome variables were previously reported for the larger sample in the original study (Tasca, et al., 2013).

Table 12 Multilevel Modelling of Change in Overall Defensive Functioning, Depressive Symptoms, Interpersonal Problems, Self Esteem and Binge Frequency Over Treatment

\begin{tabular}{lrrcrrr}
\hline & \multicolumn{3}{c}{ Fixed Effects } & \multicolumn{4}{c}{ Random Effects } \\
\cline { 2 - 7 } & \multicolumn{1}{c}{$\beta(S E)$} & \multicolumn{1}{c}{$t$} & \multicolumn{1}{c}{$p$} & \multicolumn{1}{c}{$\sigma_{\mathrm{B}}^{2}$} & \multicolumn{1}{c}{$\sigma_{\mathrm{T}}^{2}$} & $R^{2}$ \\
\hline Overall Defensive & $.26(.05)$ & 4.74 & $<.001$ & .39 & .26 & .33 \\
Functioning & $-14.81(2.37)$ & -6.24 & $<.001$ & 53.01 & 32.24 & .38 \\
Depressive Symptoms & $-33.56(6.91)$ & -4.86 & $<.001$ & 401.09 & 219.29 & .45 \\
Interpersonal Problems & $.67(.11)$ & 5.84 & $<.001$ & .13 & .09 & .36 \\
Self-Esteem & $-17.83(1.72)$ & -10.41 & $<.001$ & 50.07 & 23.09 & .54 \\
Binge Frequency &
\end{tabular}

Note. $N=77$ for defensive functioning, $N=65$ for depressive symptoms and binge frequency, and $N=63$ for interpersonal problems and self-esteem. Lower $N$ were the result of missing data. Overall Defensive Functioning (ODF) slope coefficients were based on a linear model. Depressive symptoms, Interpersonal Problems, Self Esteem, and Binge Frequency slope values were based on a loglinear modeling of time. $\sigma_{B}^{2}$ is the withinperson Level-1 variance component from the completely unconditional model. $\sigma_{\mathrm{T}}^{2}$ is the within-person variance component for the model with time (log or linear) specified at 
Level-1.

A 2-level MLM was used to test the fourth hypothesis that change in defensive functioning will be associated with change in depressive symptoms, interpersonal problems, self-esteem, and binge frequency. The results are presented in Table 13. The models testing the relationship between ODF scores slopes and change in each outcome were not significant and effect sizes were small.

Table 13

Change in Overall Defensive Functioning Scores Predicting Change in Treatment Outcome on Measures of Depression, Interpersonal Problems, Self Esteem, and Binge Frequency.

\begin{tabular}{lcrrrrr}
\hline & \multicolumn{3}{c}{ Fixed Effects } & \multicolumn{3}{c}{ Random Effects } \\
\cline { 2 - 7 } & \multicolumn{1}{c}{$\beta(S E)$} & \multicolumn{1}{c}{$t$} & \multicolumn{1}{c}{$P$} & \multicolumn{1}{c}{$\tau^{2}{ }_{\mathrm{B}}$} & \multicolumn{1}{c}{$\tau_{\mathrm{T}}^{2}$} & $R^{2}$ \\
\hline Depressive Symptoms & $.51(1.16)$ & .44 & .66 & 242.92 & 242.40 & .002 \\
Interpersonal Problems & $.48(3.43)$ & .14 & .89 & 2476.41 & 2486.39 & 00 \\
Self-Esteem & $-.03(.066)$ & -.44 & .66 & .35 & .34 & .03 \\
Binge Frequency (28 days) & $-.77(.40)$ & -1.56 & .13 & 103.36 & 101.38 & .02 \\
\hline
\end{tabular}

Note. $N=65$ for depressive symptoms and binge frequency, $N=63$ for interpersonal problems and self-esteem. Sample sizes lower than $N=77$ due to missing Level-1 data. $\tau^{2}{ }_{\mathrm{B}}$ is the between person variance component for the model controlling for pre-treatment baseline scores. $\tau^{2}{ }_{\mathrm{T}}$ is the between person variance component when ODF slope parameter is entered at Level 2 of the model.

The fifth hypothesis tested the moderating effect of attachment anxiety condition on the relationship between ODF change during group therapy and symptom outcome change from pre-treatment to 12 months post-treatment. The results indicated a small but significant moderating effect of attachment anxiety condition on the relationship between change in ODF and change in interpersonal problems. There was also a significant medium sized moderating effect of attachment 
anxiety condition on the relationship between change in ODF and change in selfesteem (Table 14). There was no significant moderating effect of attachment anxiety condition for the relationship between change in ODF and change in depressive symptoms or change in binge frequency (Table 14).

Table 14

2-Level Model Results Testing the Moderating Effect of Attachment Anxiety Condition on the Relationship Between Change in Overall Defensive Functioning and Change in Treatment Outcomes.

\begin{tabular}{llccccccc}
\hline & \multicolumn{3}{c}{ Fixed Effects } & \multicolumn{4}{c}{ Random Effects } \\
\cline { 2 - 8 } & \multicolumn{1}{c}{$\beta(S E)$} & $t$ & $d f$ & $p$ & $\tau^{2}{ }_{\mathrm{B}}$ & $\tau^{2}{ }_{\mathrm{T}}$ & $R^{2}$ \\
\hline Depressive Symptoms & $2.25(2.31)$ & 0.97 & 61 & .34 & 242.92 & 237.56 & .02 \\
Interpersonal Problems & $11.26(4.20)$ & 2.68 & 58 & .01 & 2476.41 & 2344.72 & .05 \\
Self-Esteem & $-.21(.07)$ & -3.15 & 58 & .003 & .35 & .29 & .17 \\
Binge Frequency & $1.17(.98)$ & 1.00 & 61 & .24 & 101.54 & 100.61 & .01 \\
\hline
\end{tabular}

Note. $N=65$ for Depressive Symptoms, $N=63$ for Interpersonal Problems, $N=63$ for Self-Esteem, $N=65$ for Binge Frequency. Sample sizes smaller than $N=77$ due to missing Level-1 data. $\tau^{2}{ }_{B}$ is the between person variance component for 2-Level model with baseline scores, Overall Defensive Functioning slope, and Attachment Anxiety condition entered at Level-2. $\tau^{2}{ }_{\mathrm{T}}$ is the between person variance component testing the full model with ODF slope $\mathrm{x}$ attachment anxiety interaction term at Level-2 of the model.

To explore the significant interactions, follow up analyses were conducted to evaluate the relationship between change in ODF scores and change in interpersonal problems or change in self-esteem within the high and low attachment anxiety conditions separately. For high attachment anxiety participants, greater positive change in ODF during group therapy significantly predicted improved interpersonal problems and improved self-esteem from pre-treatment to 12 months post-treatment (Table 15). Pseudo- $R^{2}$ indicated that these effects were small for interpersonal problems and medium for self-esteem. For low attachment anxiety participants, change in ODF was not 
significantly associated with change in interpersonal problems or change in self-esteem, and effects were small (Table 15).

Table 15

A 2-Level Model Testing Change in Overall Defensive Functioning and Change in Interpersonal Problems and Self-Esteem Outcomes by Treatment Condition

\begin{tabular}{lccccccccc}
\hline & & \multicolumn{3}{c}{ Fixed Effects } & \multicolumn{4}{c}{ Random Effects } \\
\cline { 3 - 10 } Variable & Condition & \multicolumn{1}{c}{$\beta(S E)$} & $t$ & $d f$ & $p$ & $\tau_{\mathrm{B}}^{2}$ & $\tau_{\mathrm{t}}^{2}$ & $R^{2}$ \\
\hline \multirow{2}{*}{$\begin{array}{l}\text { Interpersonal } \\
\text { Problems }\end{array}$} & High & $6.93(2.69)$ & 2.57 & 31 & .02 & 1878.78 & 1691.36 & .10 \\
\multirow{2}{*}{ Self-Esteem } & Low & $-4.94(3.12)$ & -1.53 & 27 & .13 & 2647.83 & 2564.66 & .03 \\
& High & -.13 & $(.04)$ & -3.24 & 31 & .01 & 0.49 & 0.42 & .14 \\
& Low & .07 & $(.06)$ & 1.16 & 27 & .26 & 0.18 & 0.16 & .05 \\
\hline
\end{tabular}

Note. $N=34$ cases for the high attachment anxiety condition. $\mathrm{N}=30$ for the low attachment anxiety condition. High and Low refer to the attachment anxiety treatment condition.

\section{Discussion}

\section{Objectives}

This study had four main objectives. The first was to evaluate if defensive functioning can be reliably assessed in a group treatment setting (i.e., meet the standard of "good" inter-rater reliability when applying DMRS coding to a group psychodynamic therapy). The second objective was to examine changes in defensive functioning during group therapy. The third was to assess if change in defensive functioning during group therapy was associated with change in treatment outcomes measured from pre-treatment to 12 months post-treatment. The fourth objective was to evaluate whether level of attachment anxiety moderated the relationship between change in defensive functioning during group therapy and change in treatment outcomes from pre-treatment to 12 months post-treatment. 


\section{Reliability Between Raters Using the DMRS}

The first hypothesis was supported. That is, the standard of "good" inter-rater reliability for the Defence Mechanism Rating Scale (DMRS) Overall Defensive Functioning (ODF) score (ICC >.60; Landis \& Koch, 1977) between raters was met. This result is consistent with previous research that used the DMRS in individual therapy that reported good to excellent reliability (Hilsenroth et al., 2003; Johansen et al., 2011; Perry \&Hogeland, 1998; Presniak et al., 2010). Achieving adequate reliability in this study was important to establish the utility of the DMRS in a group therapy setting. One of the challenges of coding the DMRS in group therapy is that with several members in each group session, the amount of time a patient can speak is limited by how much other members talk. This resulted in differences between group members in the frequency of coded defences based on how much time a patient had to speak. However, despite the variance in the frequency of coded defences per individual per session, raters in this study maintained a "good" level of reliability on average, and at similar levels to those achieved in previous research (e.g., Hilsenroth et al., 2003). These results speak to the usefulness of coding the DMRS in a group therapy setting, and to the salience of defensive functioning in understanding group therapeutic process.

Reliability between rating pairs was further tested for the more specific 7 defence levels within the overall ODF score (Table 4). Defence level reliability ranged from “poor" (Other Neurotic), to "excellent” (Major Image Disorting), with the other four defence levels falling within the "good" range (High Adaptive, Obsessional, Minor Image Distorting, Disavowal, Action) of inter-rater reliability. The results are consistent with previous research, which found that inter rater reliability was lower when assessed at 
the defence level (Hilsenroth et al., 2003, Johansen et al., 2011; Perry, et al., 2008;

Presniak, 2010). This indicates that defence level scores may be less reliable at the Other Neurotic level in group therapy, but still adequate at the other defence levels.

\section{Overall Defensive Functioning and Treatment Outcome}

Pre-treatment depression scores, interpersonal problems, self-esteem, binge frequency scores, and attachment style subscales were not significantly correlated with defensive functioning scores coded at group session three. This finding did not support the second hypothesis and is inconsistent with previous research measuring pre-treatment symptoms and defensive functioning (Hilsenroth et al., 2003; Johansen et al., 2011). However, in the current study, pre-treatment symptoms were measured up to a month before the ODF was coded in the third group therapy session. Defensive functioning may have changed at varying rates for individuals during the intervening time and during the first three sessions of the group therapy thus reducing the size of correlations. To follow up on this possibility, we conducted a post-hoc analysis by correlating defensive functioning at session 16 with post-treatment outcome measures, which occurred much closer in time. The results showed significant correlations between level of Overall Defensive Functioning and binge episodes, depressive symptoms, and attachment insecurity. This was consistent with the predicted direction of the second hypothesis, that is, lower binge eating and depressive symptoms.

\section{Change in Defensive Functioning During Group Treatment}

Defensive functioning measured at group sessions 3, 8, and 16 significantly and positively changed during treatment. The positive change in ODF scores supported the third hypothesis and was consistent with previous research that found change in defensive 
functioning in response to different types of individual therapy (Albucher et al., 1998; Hilsenroth et al., 2003, Johansen et al., 2011; Perry, et al., 2008; Presniak, 2010). This is the first time that change in defensive functioning measured by the DMRS was reported for a group treatment. The finding provides support for the validity of the DMRS when applied to Group Psychodynamic Interpersonal Psychotherapy (GPIP). Defensive functioning likely changed because of GPIP's focus on emotion regulation and on changing interpersonal patterns. According to GPIP, binge episodes occur as a result of unmet attachment needs and negative affect (Tasca et al., 2005). The negative affect leads to a defensive response (including the binge episode) to help manage negative emotions. Change in defensive functioning during GPIP may be related to the alleviation of interpersonal conflict inherent in unmet attachment needs, and improved emotion regulation. Each of these elements is associated with more adaptive relational patterns, which will have a positive effect on one's internalized self-concept (Tasca et al., 2002). Improved relationships, better affect regulations, and a positive self-concept are associated with higher level defensive functioning (Bond, 2004; Perry et al., 2009).

This study is also the first to test the change in defensive functioning during treatment for women with BED. Previous research on eating disorders has typically focused on defensive functioning and styles for patients with Anorexia Nervosa (AN) or Bulimia Nervosa $(\mathrm{BN})$. In that previous research, defensive functioning underwent a similar change to more adaptive patterns in response to individual psychological treatment (Poikolainen et al., 2001; Blaase\&Elklit, 2001). The change in defensive functioning among those with $\mathrm{AN}$ and $\mathrm{BN}$ coincided with a change in treatment outcomes, specifically a reduction in stress, an increase in self-esteem, and improved 
coping (Blaase\&Elklit, 2001). One study found a significant correlation between fewer symptoms at post-treatment and adaptive defensive style, a finding that is consistent with the post hoc correlation analysis described above in the current study (Blaase\&Elklit, 2001).

\section{Change in Defensive Functioning Associated with Change in Treatment Outcomes}

As indicated defensive functioning changed during group treatment, and this sample also showed positive and significant change in symptoms (see also, Tasca et al., 2013). However, change in overall defensive functioning was not associated with change in symptoms in this sample. This result did not support the fourth hypothesis.

Furthermore, this result is not consistent with previous research on individual therapy that found that change in ODF scores during treatment predicted better symptom outcomes up to two years post-treatment (Johansen et al., 2011). There are several possible reasons why the current study failed to find similar results. First, the sample characteristics (i.e., women with BED) differed from other studies that were conducted with mixed gender populations with a personality disorders (Hilsenroth et al., 2003; Johansen et al., 2011; Presniak et al., 2010; Perry et al., 1998). Second, the group context of the therapy might be sufficiently different from an individual therapy context to affect the nature of the defensive functioning - outcome relationship. Third, the current study's design involved creating homogeneous groups based on level of attachment anxiety. Given the potential relationship between attachment style and defensive functioning (Mikiluncer\& Shaver, 2007), it is possible that level of attachment anxiety may moderate the relationship between change in defensive functioning and change in outcomes. 


\section{Change in Defensive Functioning and Change in Treatment Outcomes Moderated}

\section{by Attachment Anxiety.}

A moderation analysis found that attachment anxiety condition did moderate the relationship between change in overall defensive functioning during group therapy and change in two of the four outcome variables one year post-treatment. This finding partially supported the fifth hypothesis. Specifically, there was a significant relationship between improved defensive functioning and improved interpersonal problems and selfesteem, but only for the high attachment anxiety condition. No association was found for the low attachment anxiety condition. The results suggest that change in defensive functioning during treatment was more meaningful for the long term interpersonal and self esteem outcomes of women with BED and higher attachment anxiety compared to those with lower attachment anxiety.

Self-esteem, interpersonal problems, and defensive functioning for those in the high attachment anxiety condition may be related to aspects of GPIP that specifically target attachment insecurity. Previous research comparing the effectiveness of GPIP to Group Cognitive Behaviour Therapy (GCBT) found differences in outcomes based on attachment anxiety (Tasca et al., 2006). Specifically, patients high in attachment anxiety benefited more from GPIP's focus on affect regulation and interpersonal problems, two concepts that can elicit a defensive response. By GPIP's focus on managing affect and improving interpersonal relationship patterns, the defensive functioning of those with high attachment anxiety can improve either through directly confronting the use of a lower level defence or by reducing the negative affect that may result in the use of a maladaptive defence. 
Further, the moderating effect of attachment anxiety on the change in defensive functioning - outcome relationship is consistent with findings from a recent study by Tasca et al. (2013). That study found that increases in therapeutic alliance (i.e. patient and therapist bond and agreement on tasks and goals) during GPIP predicted better binge eating outcomes, but only in the high attachment anxiety condition. The results of the two studies suggest that patients high in attachment anxiety may require growth in both the quality of group therapy relationships and in their own defensive functioning in order to better manage their symptoms in the long term.

\section{Clinical Implications}

The findings support the use of the DMRS as a potentially useful clinical tool to evaluate individual defensive functioning in a group context. That is, if a therapist identifies the use of lower level defenses among some patients in group, but higher level defenses among others, then the therapist might tailor interventions to the individual defenses. For example, patients with lower level defenses like Acting Out may require interventions to help them contain or control their affective experiences, whereas those with higher level defenses such as Intellectualization my require interventions to help them focus on their emotional experiences.

Furthermore, understanding the function of specific defences can assist a therapist when creating a treatment plan for patients. Defence mechanisms are a framework for understanding complex emotional processes and their regulation. Being able to identify and label that process in a therapeutic setting can be beneficial for both therapist and patient. For example, a group therapist who identifies that a patient is engaging in Passive Aggression as a defence can use that knowledge to avoid engaging in negative counter- 
transference (Bond, 2004), or to help other group members not to engage in scapegoating or counter-hostility toward the patient (Yalom\&Leszcz, 2005).

The change in defensive functioning during group therapy, and its association to changes interpersonal problems and self-esteem 12 months post-treatment for those with high attachment anxiety is consistent with the Interpersonal Model (Ansell et al., 2012) and the Psychodynamic-Interpersonal Model of BED (Tasca et al., 2002). In the Interpersonal Model of BED, relationship problems lead to negative affect, which in turn elicits a binge episode. The psychodynamic-interpersonal view put forth in the GPIP manual posits that binge episodes are potentially defensive responses to negative affect related to unmet attachment or relational needs (Tasca et al., 2002). Defensive functioning of women with BED and high attachment anxiety in the current study changed in response to GPIP, and that change was likely indicative of better internalized relationships and better coping with negative affect. Such improved relational and affective functioning likely led to fewer interpersonal problems and better self-esteem up to one-year post GPIP.

\section{Limitations}

This study had several limitations. The first is the small sample size. Of the 102 participants who began treatment, only 77 had enough coded defences $(n=>5)$ to be included in the analyses. There was a relatively large amount of missing ODF data at sessions 3,8 , and 16 , and for each outcome variable at 6-months and 12-months posttreatment. Missing pattern analyses indicated that the outcome data were MAR for outcome variables, and the missing data pattern was controlled in analyses of the ODF, hence the results are likely reliable.Nevertheless, the findings should be interpreted with 
caution, since the resulting sample size reduced statistical power and precision of the standard errors.

Another limitation was the wide range in the frequency of coded defence mechanisms per person. This issue may be specific to a group therapy setting in which individuals spoke to varying degrees across sessions. Despite the range in frequency of coded defences, we still obtained an inter-rater reliability coefficient of .70 when at least 5 defences per person were coded. This supports using the DMRS reliably in a group therapy setting. Regardless, future research should carefully consider the impact on reliability and validity of rating fewer defences per person.

The current study was based on three therapy sessions per group. This was done to balance the time constraints of the study with the goal of testing change during treatment. Training for and using the DMRS to assess therapy sessions is time consuming, but future research would greatly benefit by including more group therapy sessions for several reasons. The first is to increase the likelihood of capturing more participants engaging and discussing in the therapy. This will lead to a higher frequency of defences coded per person and reduce the impact of lower sample size. The second is to better understand the direction and nature of change in defensive functioning. The current study found that a positive linear trend best fit the data. However, by examining visual plots of ODF scores over time it appears that several scores decreased at session 8 and increased again at session 16. By including more sessions a non-linear trend of the DMRS data over time could be tested, thus providing more information to clinicians and therapists about the trajectory of change in defensive functioning. Finally, increasing the 
number of sessions would result in smaller standard errors of slope parameters, and thus increase power for certain analyses.

The participants in the study were assigned to one of two conditions based on level of attachment anxiety. This created homogenous groups in two conditions, one high in attachment anxiety and one low in attachment anxiety. This design tested the effectiveness of GPIP based on level of attachment anxiety for women with BED (Tasca et al., 2013). The homogenous nature of the groups presents several challenges when generalizing to a clinical population (see also Tasca et al., 2013). First, participants in the two conditions may not be sufficiently different to assess for differences in the outcome measures. However, the current study did find significant moderating effects of study condition on the defensive functioning - outcome relationship. That is, the study conditions appeared to differentiate individuals in a meaningful way along the dimension of attachment anxiety. Second, the results assessing differences in the attachment anxiety conditions do not specifically speak to those with secure or avoidant attachment styles. Correlation analyses were conducted on session 3 ODF scores and pre-treatment subscales of the ASQ, as well as session 16 ODF scores and post-treatment subscales of the ASQ. Only the post-treatment Confidence in Relationships subscale was significantly negatively correlated with ODF scores. The Confidence in Relationships subscale is an indicator of secure attachment, and so this finding suggests that defensive functioning may be negatively associated with attachment security, which is inconsistent with the second hypothesis. Future research is necessary to replicate this finding. Third, groups in clinical practice commonly are heterogeneously composed, and so the findings of this study may not generalize to those with high attachment anxiety in a heterogeneous group. 
Future research is required to test this moderation hypothesis in a heterogeneously composed therapy group.

\section{Generalizability and Future Directions}

Future research should include a more diverse range of participants in group treatments. The current study participants were predominantly middle-aged CanadianEuropean women with BED. Expanding the scope of future treatment studies to include other eating disorders, other mental illnesses, or individuals with diverse characteristics would increase the generalizability of the results. More importantly greater diversity in the samples would fill in the gaps in knowledge that currently exist about the defensive styles and functioning of patients with different characteristics. The current study used a self-report measure of attachment (ASQ), which likely tested just the consciously available representations of attachment. Future researchers are encouraged to assess attachment using different measures such as the Adult Attachment Interview (AAI; George, Kaplan, \& Main, 1985), which is purported to measure more unconscious aspects of attachment states of mind.

Future research should consider whether group dynamics and processesmight influence individual defensive style, and whether defences can be assessed at the group level. Of particular interest would be whether group level interactions and group level defences are related to individual level defences. This would provide information regarding how patients react to each other and how they, as a group, respond to the therapist. 


\section{Conclusion}

This study was the first to apply the DMRS to a group therapy setting, the first to examine change in defensive functioning in a sample of women with BED, and the first to test if change in defensive functioning and its effects on long term treatment outcomes was moderated by level of attachment anxiety. The DMRS can be reliably coded in a group therapy setting even when the number of coded defences per person varies. The results support the effectiveness of GPIP in not only reducing symptom severity, but in increasing adaptive defensive functioning in women with BED. Women with BED and greater attachment anxiety may require that their lower level defensive functioning improve in order for them to benefit from group psychological treatment in the long term. 


\section{References}

Albucher, R. C., Abelson, J. L., \&Nesse, R. M. (1998). Defense mechanism changes in successfully treated patients with obsessive-compulsive disorder. The American Journal of Psychiatry, 155, 558-559

American Psychiatric Association. (2000). Diagnostic and statistical manual of mental disorders (4th ed. Text rev.). Washington, DC: Author.

American Psychiatric Association. (2013). Diagnostic and statistical manual of mental disorders (Fifth ed.). Arlington, VA: American Psychiatric Publishing.

Andrews, G., Singh, M., Bond, M. (1993). The defense style questionnaire. The Journal ofNervous and Mental Disease, 181, 246-256.

Ansell, E. B., Grilo, C. M., \& White, M. A. (2012). Examining the interpersonal model of binge eating and loss of control over eating in women. International Journal of Eating Disorders, 45, 43-50.doi:10.1002/eat.20897

Bakermans-Kranenburg, M., \& van IJzendoorn, M. H. (2009). The first 10,000 adult attachment interviews: Distributions of adult attachment representations in clinical and non-clinical groups. Attachment \& Human Development, 11, 223263.doi:10.1080/14616730902814762

Bartko, J.J. (1976). On various intraclass correlation reliability coefficients.Psychological Bulletin, 83, 762-765.

Bartko, J.J. \& Carpenter, W.T. (1976). On the methods and theory of reliability. The Journal of Nervous and Mental Disease, 163, 307-317.

Beck, A. T., Steer, R. A., \& Brown, G. K. (1996).BDI-II Manual (2nd ed.). San Antonio, TX: The Psychological Corporation. 
Blaase, H., \&Elklit, A. (2001). Psychological characteristics of women with eating disorders: permanent or transient features? Scandinavian Journal of Psychology, 42, 467-478.doi:10.1111/1467-9450.00260

Bond, M., Perry, J.C., Gautier, M., Goldenberg, M., Oppenheimer, J., \&Simand, J., (1989). Validating the self-report of defense styles. Journal of Personality Disorders, 3, 101-112.

Bond, M. (2004) Empirical studies of defense style: relationships with psychopathology and change. Harvard Rev Psychiatry, 12, 263-278.doi:

$10.1080 / 10673220490886167$

Bulik, C. M., Sullivan, P. F., \&Kendler, K. S. (2002). Medical and psychiatric morbidity in obese women with and without binge eating. International Journal of Eating Disorders, 32, 72-78.doi:10.1002/eat.10072

Clark, L. A., \& Watson, D. (1995). Constructing validity: Basic issues in objective scale development. Psychological Assessment, 7, 309-319.doi:10.1037/10403590.7.3.309

Cramer, P. (1998). Coping and defense mechanisms: What's the difference? Journal of Personality, 66, 919-946.doi: 10.1111/1467-6494.00037

Cramer, P. (2007). Longitudinal study of defense mechanisms: Late childhood to late adolescence. Journal of Personality, 75, 1-23.doi: 10.1111/j.14676494.2006.00430.x

Cramer, P. (2012). Psychological maturity and change in adult defense mechanisms. Journal of Research in Personality, 46, 306-316.doi:10.1016/j.jrp.2012.02.011 
Cooper, S.H. (1998). Changing notions of defense within psychoanalytic theory. Journal of Personality, 66(6), 947-964.

Cooper, Z., \& Fairburn, C. (1987). The eating disorder examination: A semi-structured interview for the assessment of the specific psychopathology of eating disorders. International Journal of Eating Disorders, 6, 1-8.doi: 10.1002/1098-108X(198701)

Davidson, K., \&MacGregor, M.W. (1998). A critical appraisal of self-report defense mechanism measures.Journal of Personality, 66, 965-992.doi:10.1111/14676494.00039

Drapeau, M., de Roten, Y., Blake, E., Beretta, V., Strack, M., Körner, A., \&Despland, J. (2011).Defensive flexibility and its relation to symptom severity, depression, and anxiety.Journal of Nervous and Mental Disease, 199, 38-41.doi:

10.1097/NMD.0b013e3182043b4e

Enders, C.K., \&Tofighi, D. (2007). Centering predictor variables in cross-sectional multilevel models: A new look at an old issue. Psychological Methods, 12, 121138.doi: 10.1037/1082-989X.12.2.121

Fairburn, C.G. (1995). Over coming binge eating. New York, NY: The Guilford Press.

Fairburn, C. G, Cooper, Z. (1993). The Eating Disorder Examination (twelfth edition). In: C.G. Fairburn \& G.T. Wilson (eds.). Binge eating: Nature, assessment and treatment. (pp. 317-360). New York L Guilford Press, 1993.

Feeney, J. A., Noller, P., \&Hanrahan, M. (1994). Assessing adult attachment. In M. B. Sperling\& W. H. Berman (Eds.), Attachment in adults (pp.128-151). New York: Guilford Press.

Freud, A. (1966). The ego and the mechanism of defence. New York: International Press. 
(Original Work Published 1936).

Gallop, R., \&Tasca, G. A. (2009). Multilevel modeling of longitudinal data for psychotherapy researchers: II. The complexities. Psychotherapy Research, 19(45), 438-452.doi: 10.1080/10503300902849475

George, C., Kaplan, N., \& Main, M. (1985). Adult Attachment Interview.Unpublished manuscript, University of California, Berkeley.

Haan, N. (1963). Proposed model of ego functioning: Coping and defense mechanisms in relationship to IQ change. Psychological Monographs: General and Applied, 77, 1-23. doi:10.1037/h0093848

Hazan, C., \& Shaver, P. (1987). Romantic love conceptualized as an attachment process. Journal of Personality and Social Psychology, 52, 511524.doi:10.1037/0022-3514.52.3.511

Heldt, E., Blaya, C., Kipper, L., Salum, G. A., Otto, M. W., \&Manfro, G. G. (2007). Defense mechanisms after brief cognitive-behavior group therapy for panic disorder: One-year follow-up. Journal of Nervous and Mental Disease, 195, 540543.doi:10.1097/NMD.0b013e318064e7c4

Hilsenroth, M. J., Callahan, K. L., \&Eudell, E. M. (2003). Further reliability, convergent and discriminant validity of overall defensive functioning. Journal of Nervous and Mental Disease, 191, 730-737.doi:10.1097/01.nmd.0000095125.92493.e8

Høglend, P., \& Perry, J. C. (1998). Defensive functioning predicts improvement in major depressive episodes. Journal of Nervous and Mental Disease, 186, 238-243.doi: 10.1097/00005053-199804000-00006

Horowitz, L. M., Rosenberg, S. E., Baer, B. A., Ureno, G., \&Villasenor, V. S. (1988). 
Inventory of interpersonal problems: Psychometric properties and clinical applications. Journal of Consulting and Clinical Psychology, 56, 885-892. doi:10.1037/0022-006X.56.6.885

Illing, V., Tasca, G. A., Balfour, L., \&Bissada, H. (2010). Attachment insecurity predicts eating disorder symptoms and treatment outcomes in a clinical sample of women. Journal of Nervous and Mental Disease, 198, 653-659.doi:10.1097/ NMD.0b013e3181ef34b2

Johansen, P., Krebs, T. S., Svartberg, M., Stiles, T. C., \&Holen, A. (2011). Change in defense mechanisms during short-term dynamic and cognitive therapy in patients with cluster C personality disorders. Journal of Nervous and Mental Disease, 199, 712-715.doi:10.1097/NMD.0b013e318229d6a7

Kenny, D.A., Kashy, D., \& Bolger, N. (1998). Data analysis in social psychology. In D. Gilbert, S. Fiske, \& G. Lindzey (Eds.), Handbook of social psychology (4th ed., pp. 233-265). New York: McGraw-Hill.

Koskina, N., \&Giovazolias, T. (2010). The effect of attachment insecurity in the development of eating disorder disturbances across gender: The role of body dissatisfaction. The Journal of Psychology , 144, 449-471.

doi:10.1080/00223980.2010.496651

Maxwell, H., Tasca, G.A., Gick, M., Ritchie, K., Balfour, L., Bissada, H. (2012).The impact of attachment anxiety on interpersonal complementarity in early group therapy interactions among women with binge eating disorder.Group Dynamics Theory, Research, and Practice, 16, 225-271. doi: 10.1037/a0029464 
Maxwell, H., Tasca, G.A., Ritchie, K., Balfour, L., \&Bissada. H. (2013). Change in attachment insecurity is related to improved outcomes 1 year post group therapy in women with binge eating disorder. Psychotherapy, doi:10.1037/a0031100

Mikulincer, M., \& Shaver, P.R. (2007). Attachment group related processes and psychotherapy. International Journal of Group Psychotherapy.57, 233-245.doi: 10.1521/ijgp.2007.57.2.233

Mullen, L. S., Blanco, C., Vaughan, S. C., Vaughan, R., \&Roose, S. P. (1999). Defense mechanisms and personality in depression.Depression and Anxiety (Hoboken), 10, 168-174.

Olmsted, M.P., Isaacs, P., Bemis, K., \& Garner, D.M. (1988). Tape rating instrument for psychotherapy of eating disorders (TRIPED). Unpublished manuscript.

Olson, T. R., Perry, J. C., Janzen, J. I., Petraglia, J., \&Presniak, M. D. (2011). Addressing and interpreting defense mechanisms in psychotherapy: General considerations. Psychiatry, 74, 142-65.doi:http://dx.doi.org/10.1521/psyc.2011.74.2.142

Perry, J. C. (1990). The Defense Mechanism Rating Scales (5th ed.). Cambridge, MA: Cambridge Hospital.

Perry, J. C., \&Ianni, F.F. (1998).Observer-rated measures of defense mechanisms.Journal of Personality, 66, 993-1024.doi:10.1111/1467-6494.00040

Perry, J. C., \& Cooper, S.H. (1989). An empirical study of defense mechanisms: Clinical interview and life vignette ratings. Archives of General Psychiatry, 46, 444-452.

Perry, J. C., \&Høglend, P. (1998).Convergent and discriminant validity of overall defensive functioning.Journal of Nervous and Mental Disease, 186(9), 529535.doi:10.1097/00005053-199809000-00003 
Perry, J. C., Høglend, P., Shear, K., Vaillant, G. E., Horowitz, M., Kardos, M. E., . . Kagan, D. (1998). Field trial of a diagnostic axis for defense mechanisms for DSM-IV. Journal of Personality Disorders, 12, 56-

68.doi:10.1521/pedi.1998.12.1.56

Presniak, M.D., Olson, T.R., \&MacGregor, W.M. (2009). The role of defense mechanisms in borderline and antisocial personalities.Journal of Personality Assessment, 92(2), 137-145.doi: 10.1080/00223890903510373.

Presniak, M.D., Olson, T.R., Porcerelli, J.H., Dauphin, V.B. (2010). Changes in defensive functioning in a case of avoidant personality disorder. Psychotherapy: Theory, Research, Practice, Training, 47, 134-139. doi: $10.1037 / \mathrm{a} 0018838$

Perry, J.C. et al., (2009) in L, Raymond \& A. J. Stuart (Eds), Handbook of evidencebased psychodynamic psychotherapy: bridging the gap between science and practice, p. 125. Totowa, N.J.: London: Humana; Springer

Pokolainen, K., Kanerva, R., Marttunen, M., \&Lonnqvist, J. (2001). Defense styles and other risk factors for eating disorders among female adolescents: A case-control study. European Eating Disorders Review, 9, 325-334.doi: 10.1002/erv.407

Raudenbush, S. W., \&Bryk, A. S. (2002). Hierarchical linear models (2 ${ }^{\text {nd }}$ ed.). Thousand Oaks, CA: Sage Publications.

Rosenberg, M. (1965). Society and the adolescent self-image. Princeton, NJ: Princeton University Press.

Shaver, P. R., \&Mikulincer, M. (2002). Attachment-related psychodynamics. Attachment \& Human Development, 4, 133-161.doi:10.1080/14616730210154171 
Shrout, P.E., \& Fleiss, J.L. (1979). Intraclass correlations: uses in assessing rater reliability. Psychological bulletin, 86, 420-248.

Stein, D., Bronstein, Y., \&Weizman, A. (2003). Defense mechanisms in a communitybased sample of female adolescents with partial eating disorders. International Journal of Psychiatry in Medicine, 33, 343-55.doi: 10.2190/JEE0-B7PF-TVPNLJEU

Striegel-Moore, R., Wilson, G. T., Wilfley, D. E., Elder, K. A., \& Brownell, K. D. (1998). Binge eating in an obese community sample. International Journal of Eating Disorders, 23, 27-37.doi: 10.1002/(SICI)1098108X(199801)23:1<27::AID-EAT4>3.0.CO;2-3

Striegel-Moore, R., Cachelin, F. M., Dohm, F. A., Pike, K. M., Wilfley, D. E., \& Fairburn, C. G. (2001). Comparison of binge eating disorder and bulimia nervosa in a community sample. The International Journal of Eating Disorders, 29, 157165.doi: 10.1002/1098-108X(200103)29:2<157::AID-EAT1005>3.0.CO;2-8

Suldo, S. M., \& Sandberg, D. A. (2000). Relationship between attachment styles and eating disorder symptomatology among college women.Journal of College Student Psychotherapy, 15, 59-73.doi:10.1300/J035v15n01_07

Tasca, G. A., \& Gallop, R. (2009). Multilevel modeling of longitudinal data for psychotherapy researchers: I. The basics. Psychotherapy Research, 19, 429437.doi:10.1080/105033008 02641444

Tasca, G.A., Mikail, S., \& Hewitt, L.P. (2002). Group psychodynamic interpersonal psychotherapy: A manual for time limited treatment of Binge Eating Disorder. Unpublished manuscript. 
Tasca, G.A., Mikail, F.S., \& Hewitt, L.P. (2005). Group psychodynamic interpersonal psychotherapy: Summary of a treatment model and outcomes for depressive symptoms. In M. E. Abelian (Ed.), Focus on psychotherapy research (pp. 159188). New York, NY: Nova Science Publishers.

Tasca, G.A., Illing, V., Ogrodniczuk, J.S., \& Joyce, A.S. (2009). Assessing and adjusting for dependent observations in group treatment research using multilevel models. Group Dynamics: Theory, Research, and Practice, 13, 151-162. doi: $10.1037 / \mathrm{a} 0014837$

Tasca, G.A., Ritchie, K., \& Balfour, L. (2011). Implications of attachment theory and research for the assessment and treatment of eating disorders.Psychotherapy, 48 , 249-259.doi: 10.1037/a0022423

Tasca, G. A., Ritchie, K., Demidenko, N., Balfour, L., Krysanski, V., Weekes, K., Barber, A., Keating, L., \&Bissada, H. (2013). Matching women with binge eating disorder to group treatment based on attachment anxiety: Outcomes and moderating effects. Psychotherapy Research, 23, 301-314.doi:

$10.1080 / 10503307.2012 .717309$

Tasca, G.A., Ritchie, K., Conrad, G., Balfour, L., Gayton, J, Lybanon, L., \&Bissada, H. (2006): Attachment scales predict outcome in a randomized controlled trial of two group therapies for binge eating disorder:An aptitude by treatment interaction. Psychotherapy Research, 16, 106-121.doi: 10.1080/10503300500090928

Troisi, A., D’Argenio, A., Peracchio, F., \& Petti, P. (2001).Insecure attachment and alexithymia in young men with mood symptoms.The Journal of Nervous and Mental Disease, 189, 311-316.doi:10.1097/00005053-200105000-00007 
Vaillant, G.E. (1977). Adaptation to life. Boston: Little, Brown.

Vaillant, G.E. (1994). Ego mechanisms of defense and personality psychopathology.Journal of Abnormal Psychology, 103, 44-50.doi:

10.1037/0021-843X.103.1.44

Vocks, S., Tuschen-Caffier, B., Pietrowsky, R., Rustenbach, S.J., Kersting, A., \&Herpertz, S. (2010). Meta-analysis of the effectiveness of psychological and pharmacological treatments for binge eating disorder.International Journal of Eating Disorders, 43, 205-217.doi: 10.1002/eat.20696

Waters, E., Hamilton, C. E., \&Weinfield, N. S. (2000a). The stability of attachment security from infancy to adolescence and early adulthood: General introduction. Child Development, 71, 678-683.

Waters, E., Merrick, S., Treboux, D., Crowell, J., \&Albersheim, L. (2000b) Attachment security in infancy and early adulthood: A twenty-year longitudinal study. Child development, 71, 684-689. doi:10.111/1467-8624.00176

Yalom, I. D., \&Leszcz, M. (2005). The theory and practice of group psychotherapy (5th ed.). New York: Basic Books. 
Appendix A:

\section{Defence Mechanism Rating Scale Scoring Sheet}

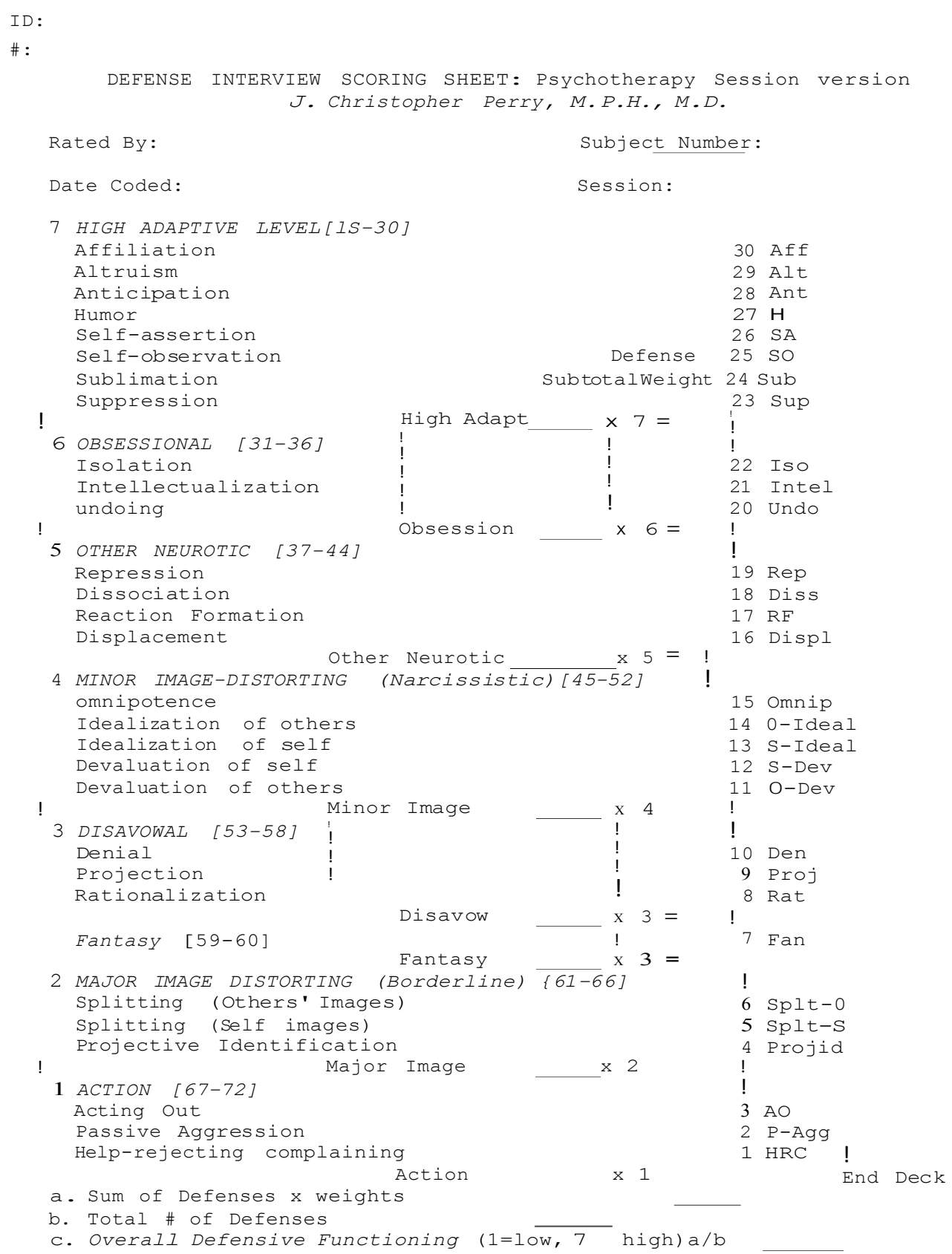


Appendix B:

DSM-IV-TR Diagnostic Criteria for Binge-Eating Disorder (APA, 2000, p. 787)

A. Recurrent episodes of binge eating. An episode is characterized by the following:

1) Eating an unusually large amount of food in a discrete period of time, and

2) A sense of lost control over eating during the episode of binge eating

B. Binge episodes are associated with three or more of the following:

1) Eating more rapidly than usual

2) Feeling uncomfortably full from episodes of binge eating

3) Eating large quantities of food when one does not physically feel hungry

4) Eating alone due to feelings of embarrassment regarding one's food intake

5) Feelings of depression, , or disgust with oneself after binge eating episodes

C. Distress over episodes of binge eating is present.

D. Binge eating episodes occur at least, on average, two days a week for six months. 
Appendix C

Attachment Style Questionnaire (ASQ)

\section{Appendix 5.1. Attachment Style Questionnaire}

Show how much you agree with each of the following items by rating them on this scale: 1 - totally disagree; 2 - strongly disagree; 3 - slightly disagree; 4 - slightly agree; 5 - strongly agree; or 6 - totally agree.

Confidence

Confidence

Confidence

Discomfort

Discomfort

$R$ as $S$

$R$ as $S$

$\mathrm{R}$ as $\mathrm{S}$

$\mathrm{R}$ as $\mathrm{S}$

$R$ as $S$

$\mathrm{N}$ for $\mathrm{A}$

$\mathrm{N}$ for $\mathrm{A}$

$\mathrm{N}$ for $\mathrm{A}$

$R$ as $S$

$\mathrm{N}$ for $\mathrm{A}$

Discomfort

Discomfort

Preoccupation

Confidence

Discomfort

Discomfort

Preoccupation

Discomfort

$\mathrm{N}$ for $\mathrm{A}$

Discomfort

Discomfort

$\mathrm{N}$ for $\mathrm{A}$
1. Overall, 1 am a worthwhile person.

2. I am easier to get to know than most people.

3. I feel confident that other people will be there for me when I need them.

4. 1 prefer to depend on myself rather than other people.

5. 1 prefer to keep to myself.

6. To ask for help is to admit that you're a failure.

7. People's worth should be judged by what they achieve.

8. Achieving things is more important than building relationships.

9. Doing your best is more important than getting on with others

10. If you've got a job to do, you should do it no matter who gets hurt.

11. It's important to me that others like me.

12. It's important to me to avoid doing things that others won't like.

13. I find it hard to make a decision unless I know what other people think.

14. My relationships with others are generally superficial.

15. Sometimes 1 think 1 am no good at all.

16. I find it hard to trust other people.

17. 1 find it difficult to depend on others.

18. I find that others are reluctant to get as close as I would like.

19. I find it relatively easy to get close to other people.

20. I find it easy to trust others. (R)

21. I feel comfortable depending on other people. (R)

22. I worry that others won't care about me as much as I care about them.

23. I worry about people getting too close.

24. I worry that 1 won't measure up to other people.

25. I have mixed feelings about being close to others.

26. While I want to get close to others, I feel uneasy about it.

27. I wonder why people would want to be involved with me. 
Appendix D

Beck Depression Inventory

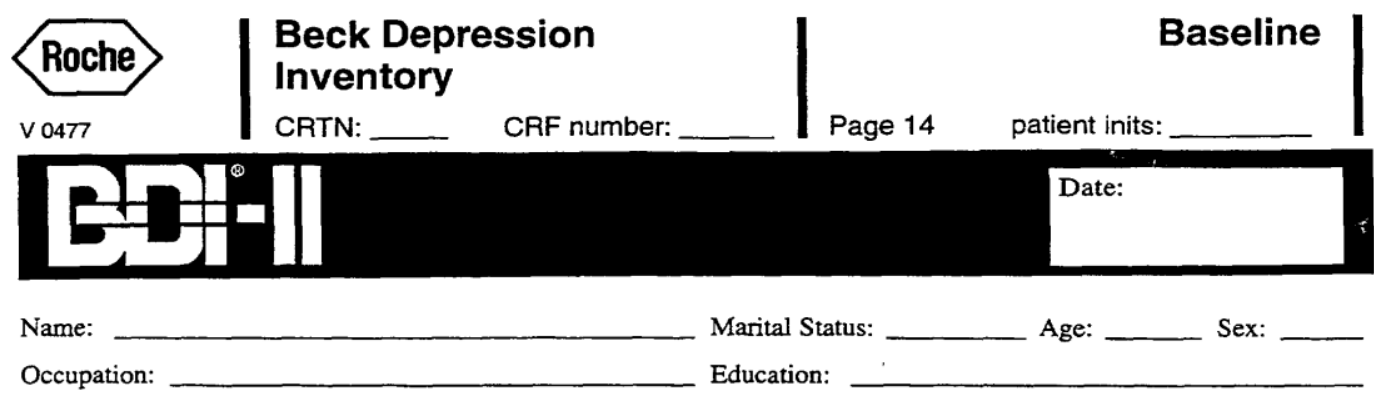

Instructions: This questionnaire consists of 21 groups of statements. Please read each group of statements carefully, and then pick out the one statement in each group that best describes the way you have been feeling during the past two weeks, including today. Circle the number beside the statement you have picked. If several statements in the group seem to apply equally well, circle the highest number for that group. Be sure that you do not choose more than one statement for any group, including Item 16 (Changes in Sleeping Pattern) or Item 18 (Changes in Appetite).

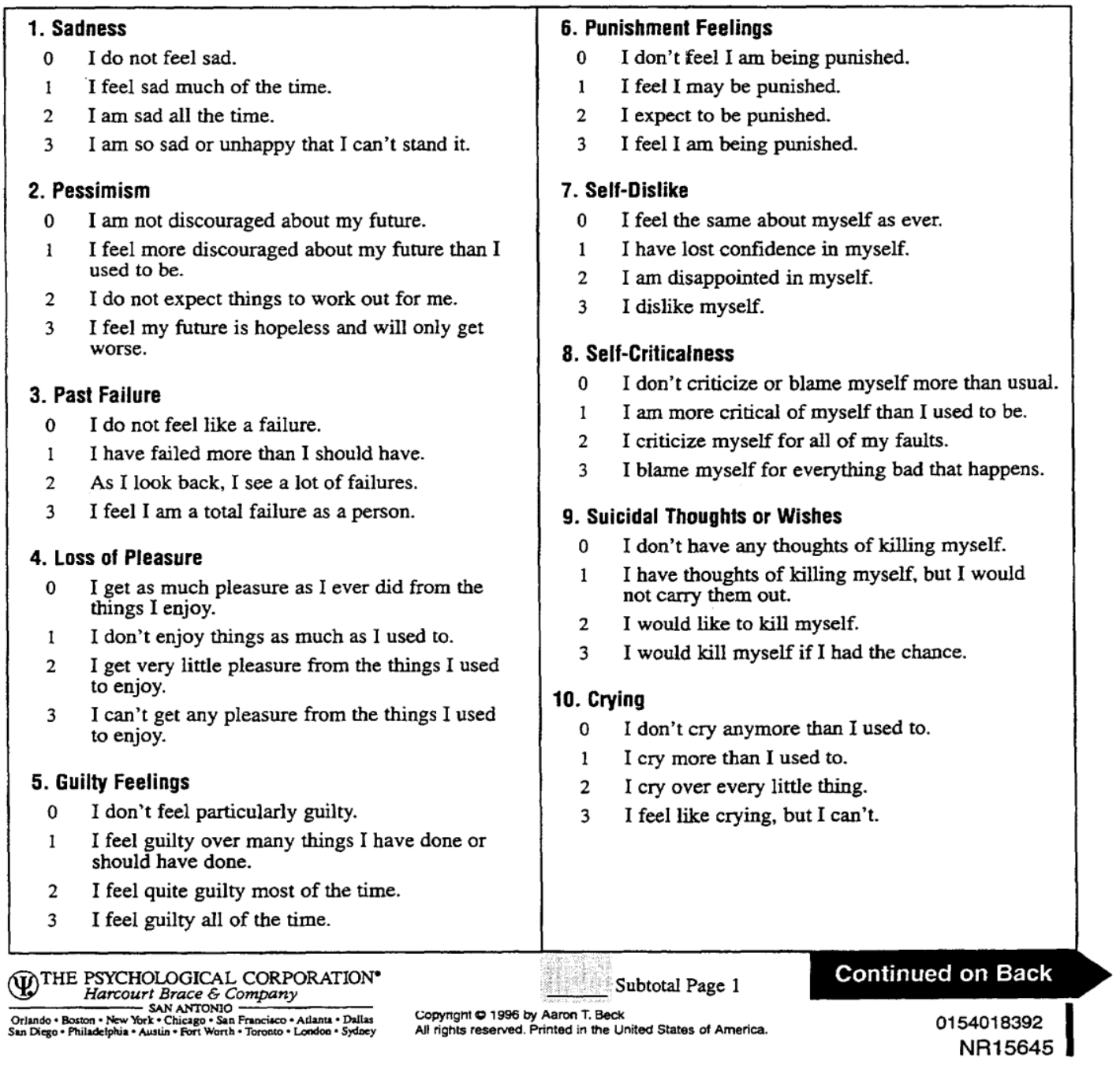




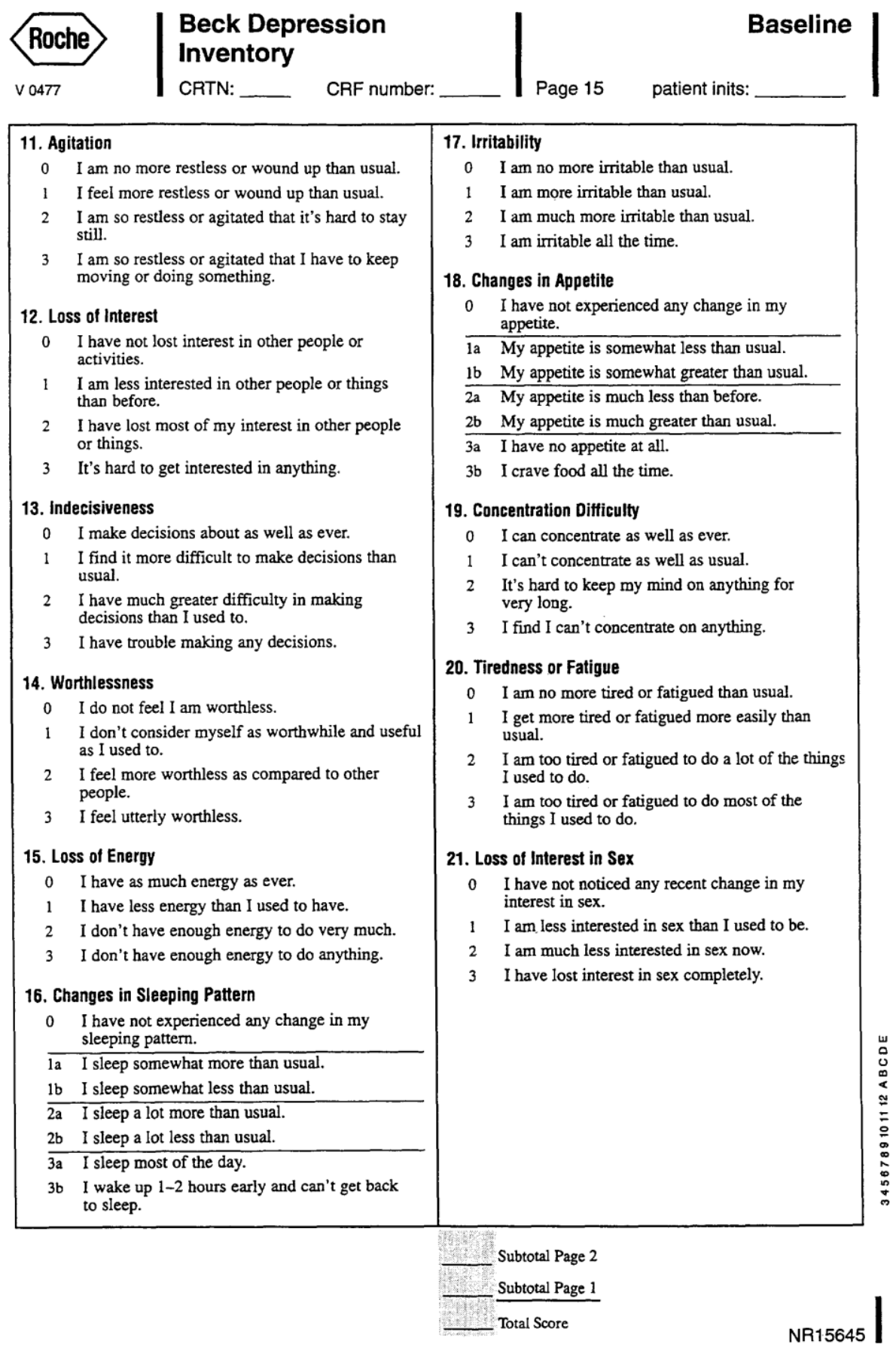




\section{Appendix E}

\section{Inventory of Interpersonal Problems}

\section{IIP-64.}

Name:

Date: $\frac{}{\text { Monh }} / \frac{}{\text { Doy }} / \frac{}{\text { Year }}$ Sex: Male $\square$ Female $\square$

People have reported having the following problems in relating to other people. Please read the list below, and for each item, consider whether it has been a problem for you with respect to any significant person in your life. Then fill in the numbered circle that describes how distressing that problem has been.

The following are things you find hard to do with other people.

It is hard for me to:

1. Trust other people

2. Soy "no" to other people

3. Join in on groups

4. Keep things private from other people

5. Let other people know what I want

6. Tell a person to stop bothering me

7. Introduce myself to new people

8. Confront people with problems that come up

9. Be assertive with another person

10. Let other people know when I am angry

11. Make a long-term commitment to another person

12. Be another person's boss

13. Be aggressive toward other people when the situation calls for it

14. Socialize with other people

15. Show affection to people

16. Get along with people

17. Understand another person's point of view

18. Express my feelings to other people directly

19. Be firm when I need to be

20. Experience a feeling of love for another person

21. Set limits on other people

22. Be supportive of another person's goals in life

23. Feel close to other people

24. Really care about other people's problems

25. Argue with another person

26. Spend time alone

27. Give a gift to another person

28. Let myself foel angry at somebody I like

29. Put somebody else's needs before my own

30. Stry out of other people's business

31. Take instructions from people who have authority over me

32. Feel good about another person's happiness

33. Ask other people to get together socially with me

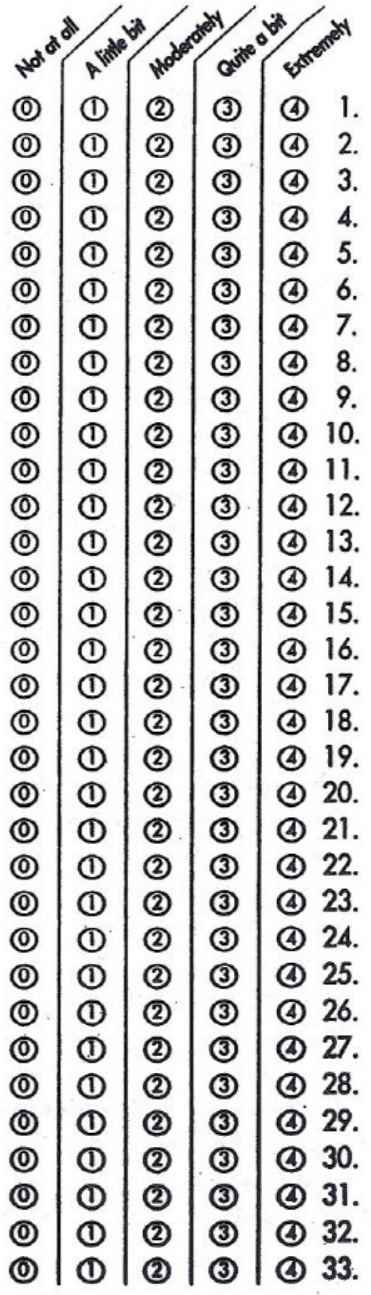


It is hard for me to:

34. Feel angry at other people

35. Open up and tell my feelings to another person

36. Forgive another person after l've been angry

37. Attend to my own welfare when somebody else is needy

38. Be assertive without worrying about hurting the other person's feelings

39. Be self-confident when I am with other people

The following are things that you do too much.

40. I fight with other people too much.

41. I feel too responsible for solving other people's problems.

42. I am too easily persuaded by other people.

43. I open up to people too much.

44. I am too independent.

45. I am too aggressive toward other people.

46. I try to please other people too much.

47. I clown around too much.

48. I want to be noticed too much.

49. I trust other people too much.

50. I try to control other people too much.

51. I put other people's needs before my own too much.

52. I try to change other people too much.

53. I am too gullible.

54. I am overly generous to other people.

55. I am too afraid of other people.

56. I am too suspicious of other people.

57. I manipulate other people too much to get what I want.

58. I tell personal things to other people too much.

59 . I argue with other people too much.

60 . I keep other people at a distance too much.

61. I let other people take advantage of me too much.

62. I feel embarrassed in front of other people too much.

63. I am affected by another person's misery too much.

64. I want to get revenge against people too much.

\begin{tabular}{|c|c|c|c|c|}
\hline & & & & \\
\hline & (1) & (2) & (3) & (4) 34 \\
\hline & (1) & (2) & (3) & (4) 35 \\
\hline & (1) & (2) & (3) & \\
\hline & (1) & (2) & (3) & \\
\hline & (1) & (2) & (3) & \\
\hline & (1) & (2) & (3) & \\
\hline & (1) & (2) & (3) & (4) 40 . \\
\hline & (1) & (2) & (3) & \\
\hline & (1) & (2) & (3) & \\
\hline & (1) & (2) & (3) & \\
\hline & (1) & (2) & (3) & \\
\hline & (1) & (2) & (3) & \\
\hline & (1) & (2) & (3) & \\
\hline & (1) & (2) & (3) & \\
\hline & (1) & (2) & (3) & \\
\hline & (1) & (2) & (3) & \\
\hline & (1) & (2) & (3) & \\
\hline & (1) & (2) & (3) & (4) 51 . \\
\hline & (1) & (2) & (3) & (4) 52 \\
\hline & (1) & (2) & (3) & (4) \\
\hline & (1) & (2) & (3) & \\
\hline & (1) & (2) & (3) & \\
\hline & (1) & (2) & (3) & \\
\hline & (1) & (2) & (3) & \\
\hline & (1) & (2) & (3) & \\
\hline & (1) & (2) & (3) & \\
\hline & (1) & (2) & (3) & (4) \\
\hline & (1) & (2) & (3) & \\
\hline & (1) & (2) & (3) & \\
\hline & (1) & (2) & (3) & \\
\hline & & & & \\
\hline
\end{tabular}




\section{Appendix F}

\section{Rosenberg Self-Esteem Scale}

DIRECTIONS: Please indicate how much you agree or disagree with the following statements. Circle the appropriate number beside each statement.

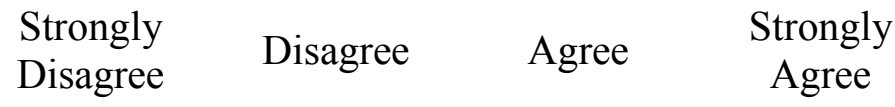

1. I feel that I am a person of worth, at least on an equal plane with others.

2. I feel that I have a number of good qualities.

3. All in all, I am inclined to feel that I am a failure.

4. I am able to do things as well as most other people.

5. I feel that I do not have much to be proud of.

6. I take a positive attitude toward myself.

7. On the whole, I am satisfied with myself.

8. I wish I could have more respect for myself.

9. I certainly feel useless at times. 


\section{Appendix G}

\section{Multi-Level Model Equations}

Model 1: 3-Level Model used to test dependence (ICC; $\rho$ ) of the linear growth model for each outcome.

Level 1: $\mathrm{y}_{\mathrm{tij}}=\pi_{0 \mathrm{ij}}+\pi_{1 \mathrm{ij}}\left(\mathrm{time}_{\mathrm{tij}}\right)+\mathrm{e}_{\mathrm{tij}}$

Level 2: $\pi_{0 \mathrm{ij}}=\beta_{00 \mathrm{j}}+\beta_{01 \mathrm{j}}($ individual baseline $)+\mathrm{r}_{0 \mathrm{ij}}$

$\pi_{1 \mathrm{ij}}=\beta_{10 \mathrm{j}}+\beta_{11 \mathrm{j}}($ individual baseline $)+\mathrm{r}_{1 \mathrm{ij}}$

Level 3: $\beta_{00 \mathrm{j}}=\gamma_{000}+\gamma_{001}($ group mean baseline $)+\gamma_{002}($ attachment condition $)+\mathrm{u}_{00 \mathrm{j}}$

$\beta_{01 \mathrm{j}}=\gamma_{010}+\mathrm{u}_{01 \mathrm{j}}$

$\beta_{10 \mathrm{j}}=\gamma_{100}+\gamma_{101}($ group mean baseline $)+\gamma_{102}($ attachment condition $)+\mathrm{u}_{10 \mathrm{j}}$

$\beta_{11 \mathrm{j}}=\gamma_{110}+\mathrm{u}_{11 \mathrm{j}}$

Individual baseline was group mean centred, and group mean baseline was grand mean centred. Time was linear for Overall Defensive Functioning (ODF) scores, and time was $\log$ transformed for other outcomes.

Model 2: 2-Level Model testing linear change in ODF scores over treatment.

Level 1: $\mathrm{y}_{\mathrm{ij}}=\pi_{0 \mathrm{ij}}+\pi_{1 \mathrm{i}}\left(\right.$ time $\left._{\mathrm{ti}}\right)+\mathrm{e}_{\mathrm{ij}}$

Level 2: $\pi_{0 \mathrm{i}}=\beta_{00}+\beta_{01}($ completer $)+\beta_{02}(\mathrm{ODF} 3)+\mathrm{r}_{0 \mathrm{i}}$ $\pi_{1 \mathrm{i}}=\beta_{10}+\beta_{11}($ completer $)+\beta_{21}(\mathrm{ODF} 3)+\mathrm{r}_{1 \mathrm{i}}$

Notes: Completer status entered as the Level-2 covariate to control for the effects of noncompletion status. ODF at session 3 was controlled as the baseline value. 
Model 3: 2-level model testing the association between change in ODF during group therapy and change in outcomes from pre-treatment to 12 month follow up.

Level 1: $Y_{\mathrm{ij}}=\pi_{0 \mathrm{i}}+\pi_{1 \mathrm{i}}\left(\operatorname{logtime}_{\mathrm{ti}}\right)+\mathrm{e}_{\mathrm{ti}}$

Level 2: $\pi_{0 \mathrm{i}}=\beta_{00}++\beta_{01}($ individual baseline $)+\beta_{02}\left(\mathrm{ODF}\right.$ slope $\left.\mathrm{ij}_{\mathrm{ij}}\right)+\mathrm{r}_{0 \mathrm{i}}$ $\pi_{1 \mathrm{i}}=\beta_{10}++\beta_{11}($ individual baseline $)+\beta_{12}\left(\right.$ ODF slope $\left.\mathrm{ij}_{\mathrm{ij}}\right)+\mathrm{r}_{1 \mathrm{i}}$

Individual baseline scores were grand mean centered at Level-2 to control for pretreatment scores. $Y_{\mathrm{ij}}$ represents the treatment outcome variables (Beck Depression Inventory, Inventory of Interpersonal Problems, Rosenberg Self-Esteem Scale, and Binge Frequency). Time was log transformed.

Model 4: 2-Level model assessing the moderating effect of attachment anxiety condition on the ODF slope by outcome relationship.

Level 1: $\mathrm{y}_{\mathrm{ij}}=\pi_{0 \mathrm{i}}+\pi_{1 \mathrm{i}}\left(\right.$ timelog $\left._{\mathrm{ti}}\right)+\mathrm{e}_{\mathrm{ij}}$

Level 2: $\pi_{0 \mathrm{i}}=\beta_{00}+\beta_{01}($ individual baseline $)+\beta_{02}\left(\mathrm{ODF}_{\text {slope }}\right)+\beta_{03}($ attachment condition $\left._{\mathrm{ij}}\right)+\beta_{04 \mathrm{j}}\left(\right.$ attachment condition $\mathrm{x}$ ODF slope $\left._{\mathrm{ij}}\right)+\mathrm{r}_{0 \mathrm{i}}$ $\pi_{1 \mathrm{ij}}=\beta_{10}+\beta_{11}($ individual baseline $)+\beta_{12}\left(\right.$ ODF slope $\left._{\mathrm{ij}}\right)+\beta_{13}\left(\right.$ attachment condition $\left._{\mathrm{ij}}\right)+$ $\beta_{14}\left(\right.$ attachment condition $x$ ODF slope $\left._{i j}\right)+r_{1 i}$

Notes: Individual baseline scores at Level-2 were grand mean centered. Condition was dummy coded $(0,1)$. ODF slope was uncentered. $\mathrm{y}_{\mathrm{ij}}$ is the outcome variable (BDI, IIP, RSES, and Binge Frequency). 